\title{
COMPOSITION OPERATORS WITHIN SINGLY GENERATED COMPOSITION $C^{*}$-ALGEBRAS
}

\author{
THOMAS L. KRIETE, BARBARA D. MACCLUER, AND JENNIFER L. MOORHOUSE
}

\begin{abstract}
Let $\varphi$ be a linear-fractional self-map of the open unit disk $\mathbb{D}$, not an automorphism, such that $\varphi(\zeta)=\eta$ for two distinct points $\zeta, \eta$ in the unit circle $\partial \mathbb{D}$. We consider the question of which composition operators lie in $C^{*}\left(C_{\varphi}, \mathcal{K}\right)$, the unital $C^{*}$-algebra generated by the composition operator $C_{\varphi}$ and the ideal $\mathcal{K}$ of compact operators, acting on the Hardy space $H^{2}$. This necessitates a companion study of the unital $C^{*}$-algebra generated by the composition operators induced by all parabolic non-automorphisms with common fixed point on the unit circle.
\end{abstract}

\section{INTRODUCTION}

Given any analytic self-map $\varphi$ of the unit disk $\mathbb{D}$ in the complex plane, one can form the composition operator $C_{\varphi}: f \rightarrow f \circ \varphi$, which acts as a bounded operator on the Hardy space $H^{2}$. This paper is the second in a series of three investigating spectral theory in $C^{*}$-algebras generated by certain composition and Toeplitz operators. In the first article [15, we studied $C^{*}\left(T_{z}, C_{\varphi}\right)$, the unital $C^{*}$ algebra generated by the unilateral shift $T_{z}$ on $H^{2}$ and a single composition operator $C_{\varphi}$ with $\varphi$ satisfying

(1)

$\{\varphi$ is a linear-fractional self-map of $\mathbb{D}$ which is not an automorphism, and $\{\varphi(\zeta)=\eta$ for distinct points $\zeta, \eta$ in the unit circle $\partial \mathbb{D}$.

Throughout the current paper, $\varphi$ will always have this meaning. The algebra $C^{*}\left(T_{z}, C_{\varphi}\right)$ necessarily contains the ideal $\mathcal{K}$ of compact operators on $H^{2}$. The main result of [15] identifies $C^{*}\left(T_{z}, C_{\varphi}\right) / \mathcal{K}$ with a certain $C^{*}$-algebra of $2 \times 2$ matrix valued functions; see Theorem 4.12 of [15. The case where $\varphi$ is replaced by an automorphism of $\mathbb{D}$, or even a discrete group of automorphisms, has been studied by Jury [12, [13, and has a rather different character.

The shift $T_{z}$ does not appear to play a role in the questions we consider in this paper; accordingly we omit it and study $C^{*}\left(C_{\varphi}, \mathcal{K}\right)$, the unital $C^{*}$-algebra generated by $C_{\varphi}$, for $\varphi$ as described above, and the compact operators. The composition $\varphi \circ \varphi$ has sup-norm strictly less than 1 , so that $C_{\varphi}^{2}=C_{\varphi \circ \varphi}$ is compact and nonzero. Since $\varphi$ has no boundary fixed point, a theorem of Guyker [10] shows that $C_{\varphi}$ is irreducible if and only if $\varphi(0) \neq 0$. It follows that when $\varphi(0) \neq 0$ the unital $C^{*}$-algebra $C^{*}\left(C_{\varphi}\right)$ generated by $C_{\varphi}$ alone contains $\mathcal{K}$; see [5], p.74. We want our $C^{*}$-algebras to always contain $\mathcal{K}$, and we indicate this by continuing to write $C^{*}\left(C_{\varphi}, \mathcal{K}\right)$ if the irreducibility criterion $\varphi(0) \neq 0$ is in doubt.

Date: July 10, 2008.

2000 Mathematics Subject Classification. Primary 47B33. 
Let $\mathcal{P}$ denote the dense subalgebra of $C^{*}\left(C_{\varphi}, \mathcal{K}\right)$ consisting of all finite linear combinations of the identity $I$, all words in $C_{\varphi}$ and $C_{\varphi}^{*}$, and all compact operators. An element $B$ in $\mathcal{P}$ has a unique representation of the form

$$
B=c I+f\left(C_{\varphi}^{*} C_{\varphi}\right)+g\left(C_{\varphi} C_{\varphi}^{*}\right)+C_{\varphi} p\left(C_{\varphi}^{*} C_{\varphi}\right)+C_{\varphi}^{*} q\left(C_{\varphi} C_{\varphi}^{*}\right)+K
$$

where $f, g, p$ and $q$ are polynomials, $f(0)=0=g(0), c$ is complex, and $K$ is compact. Let $s=1 /\left|\varphi^{\prime}(\zeta)\right|$ and write $\mathcal{D}$ for the $C^{*}$-algebra of continuous $2 \times 2$ matrix-valued functions $F$ on $[0, s]$ with $F(0)$ a scalar multiple of the identity, equipped with the supremum operator norm. It was shown in [15] that there is a unique *-homomorphism $\Psi$ of $C^{*}\left(C_{\varphi}, \mathcal{K}\right)$ onto $\mathcal{D}$ with $\operatorname{Ker} \Psi=\mathcal{K}$ and such that

$$
\Psi(B)=\left[\begin{array}{lr}
c+g & r p \\
r q & c+f
\end{array}\right]
$$

where $B$ is given by Equation (2) and $r(t)=\sqrt{t}$. Equivalently, we have a short exact sequence of $C^{*}$-algebras

$$
0 \rightarrow \mathcal{K} \stackrel{i}{\rightarrow} C^{*}\left(C_{\varphi}, \mathcal{K}\right) \stackrel{\Psi}{\rightarrow} \mathcal{D} \rightarrow 0
$$

where $i$ is inclusion. For any operator $T$ on $H^{2}$ we write $\|T\|_{e}$ for the essential norm of $T$, that is, the distance from $T$ to the ideal $\mathcal{K}$. We note that if $T$ is in $C^{*}\left(C_{\varphi}, \mathcal{K}\right)$, then $\|T\|_{e}=\|\Psi(T)\|$.

For bounded operators $A$ and $B$ on $H^{2}$, let us write $A \equiv B(\bmod \mathcal{K})$ if there exists a compact operator $K$ with $A=B+K$. In 15 the authors used C. Cowen's well-known adjoint formula [7] to show that if

$$
\psi(z)=\frac{a z+b}{c z+d}
$$

is a linear-fractional self map of $\mathbb{D}$, not an automorphism but satisfying $\left|\psi\left(z_{0}\right)\right|=1$ for some $z_{0} \in \partial \mathbb{D}$, then

$$
C_{\psi}^{*} \equiv \frac{1}{\left|\psi^{\prime}\left(z_{0}\right)\right|} C_{\sigma_{\psi}}(\bmod \mathcal{K})
$$

where $\sigma_{\psi}$ is the so-called "Krein adjoint" of $\psi$,

$$
\sigma_{\psi}(z)=\frac{\bar{a} z-\bar{c}}{-\bar{b} z+\bar{d}} .
$$

We denote the Krein adjoint of $\varphi$ itself simply by $\sigma$, which the reader can distinguish by context from the spectrum $\sigma(T)$ or essential spectrum $\sigma_{e}(T)$ of an operator $T$.

Now consider an operator $B$ in the dense subalgebra $\mathcal{P}$, as expressed in Equation (2). Since $I=C_{z}, C_{\varphi}^{*} C_{\varphi} \equiv s C_{\varphi \circ \sigma}(\bmod \mathcal{K})$ and $C_{\varphi} C_{\varphi}^{*} \equiv s C_{\sigma \circ \varphi}(\bmod \mathcal{K})$, where $s=1 /\left|\varphi^{\prime}(\zeta)\right|$, we can rewrite Equation (2) as

$$
B=c C_{z}+A_{1}+A_{2}+A_{3}+A_{4}+K^{\prime}
$$

where $K^{\prime}$ is compact and $A_{1}, A_{2}, A_{3}$, and $A_{4}$ are finite linear combinations of composition operators whose associated self-maps are chosen, respectively, from the four lists

$$
\left\{(\varphi \circ \sigma)_{n_{1}}\right\},\left\{(\sigma \circ \varphi)_{n_{2}}\right\},\left\{(\varphi \circ \sigma)_{n_{3}} \circ \varphi\right\},\left\{(\sigma \circ \varphi)_{n_{4}} \circ \sigma\right\} .
$$

Here we write $(\psi)_{n}$ for the $n^{\text {th }}$ iterate of a self-map $\psi$ of $\mathbb{D}$, and let $n_{k}$ range over the positive integers for $k=1,2$, and over the non-negative integers for $k=3,4$. See [15] for further details. Thus $C^{*}\left(C_{\varphi}, \mathcal{K}\right)$ is spanned, modulo the compacts, by 
actual composition operators. This leads to our main question: for which analytic self-maps $\psi$ of $\mathbb{D}$ does $C_{\psi}$ lie in $C^{*}\left(C_{\varphi}, \mathcal{K}\right)$ ?

In particular we will describe explicitly, in both function-theoretic and operatortheoretic terms, all linear-fractional composition operators lying in $C^{*}\left(C_{\varphi}, \mathcal{K}\right)$. This description plays a role in the third paper of our series [16 which is devoted to spectral theory in Toeplitz-composition algebras with several generators. Along the way here we show that if $C^{*}\left(\mathbb{P}_{\gamma}\right)$ denotes the unital $C^{*}$-algebra generated by composition operators induced by the parabolic non-automorphisms fixing $\gamma$ in the unit circle, then there is a short exact sequence

$$
0 \rightarrow \mathcal{K} \rightarrow C^{*}\left(\mathbb{P}_{\gamma}\right) \rightarrow C([0,1]) \rightarrow 0
$$

of $C^{*}$-algebras, where $C([0,1])$ denotes the algebra of continuous functions on the unit interval.

\section{NECESSARY CONDITIONS}

If $\psi: \mathbb{D} \rightarrow \mathbb{D}$ is analytic we write $F(\psi)$ for the set of points $\alpha$ in $\partial \mathbb{D}$ at which $\psi$ has a finite angular derivative in the sense of Caratheodory; see [8, 21. In particular, if $\alpha$ is in $F(\psi)$, the nontangential limit $\psi(\alpha)$ necessarily exists and has modulus one. We write $\psi^{\prime}(\alpha)$ for the angular derivative of $\psi$ at $\alpha$. Recall that this is the ordinary derivative if $\psi$ extends analytically to a neighborhood of $\alpha$ and $|\psi(\alpha)|=1$.

It is well known that if $C_{\psi}$ is compact on $H^{2}$, then $F(\psi)$ is empty [20. When $C_{\psi}$ is considered as acting on the Bergman space in $\mathbb{D}$, the converse assertion is true [17. On the space $H^{2}$ considered here, however, " $C_{\psi}$ is compact" is a strictly stronger requirement than " $F(\psi)$ is empty"; see, for example, [8], 21]. Our first goal is to show that when $C_{\psi}$ lies in $C^{*}\left(C_{\varphi}, \mathcal{K}\right)$ these two conditions are equivalent.

First we recall that a linear-fractional self-map $\rho$ of $\mathbb{D}$ is parabolic if $\rho$ fixes one point $\gamma$ in $\partial \mathbb{D}$ and is conjugate, via the map $(\gamma+z) /(\gamma-z)$, to translation in the right half-plane $\Omega=\{w: \operatorname{Re} w>0\}$ by a complex number $a$ with non-negative real part. We denote this parabolic map by $\rho_{a}$, or by $\rho_{\gamma, a}$ if the fixed point $\gamma$ is not clear from the context. A linear-fractional map $\rho$ with fixed point $\gamma$ in $\partial \mathbb{D}$ is parabolic provided $\rho^{\prime}(\gamma)=1$. Another representation of $\rho_{a}$ will prove useful. The map $\tau_{\gamma}(z)=i(\gamma-z) /(\gamma+z)$ carries $\mathbb{D}$ onto the upper half-plane $\{w: \operatorname{Im} w>0\}$ and takes $\gamma$ to 0 , rather than infinity. We write $u$ for the conjugate of $\rho_{a}$ by $\tau_{\gamma}$ : $u=\tau_{\gamma} \circ \rho_{a} \circ \tau_{\gamma}^{-1}$. One readily computes that $u(w)=i w /(i+w a)$, and so

$$
u^{\prime \prime}(0)=2 i a \text {. }
$$

Also important for us will be several lower bounds for the essential norm of a linear combination of composition operators. Given an analytic self-map $\psi$ of $\mathbb{D}$ and $\alpha$ in $F(\psi)$, we call $D_{1}(\psi, \alpha) \equiv\left(\psi(\alpha), \psi^{\prime}(\alpha)\right)$ the first order data vector for $\psi$ at $\alpha$. If we have a finite collection of maps $\psi_{1}, \psi_{2} \cdots, \psi_{n}$ and $\alpha$ lies in the union of the finite angular derivative sets $F\left(\psi_{1}\right), F\left(\psi_{2}\right), \cdots F\left(\psi_{n}\right)$, we define

$$
\mathcal{D}_{1}(\alpha)=\left\{D_{1}\left(\psi_{j}, \alpha\right): 1 \leq j \leq n \text { and } \alpha \in F\left(\psi_{j}\right)\right\},
$$

the set of possible first order data vectors at $\alpha$ coming from that collection of maps. Theorem 5.2 in 14] states that if $\mathcal{D}_{1}(\alpha)$ is non-empty, then for any complex numbers

$c_{1}, \cdots, c_{n}$, 


$$
\left\|c_{1} C_{\psi_{1}}+\cdots+c_{n} C_{\psi_{n}}\right\|_{e}^{2} \geq \sum_{\mathbf{d} \in \mathcal{D}_{1}(\alpha)}\left|\sum_{\substack{\alpha \in F\left(\psi_{j}\right) \\ D_{1}\left(\psi_{j}, \alpha\right)=\mathbf{d}}} c_{j}\right|^{2} \frac{1}{\left|d_{1}\right|},
$$

where $\mathbf{d}=\left(d_{0}, d_{1}\right)$.

There is a higher order version of this lower bound which works provided that for the specific $\alpha$ in $F(\psi), \psi$ is analytically continuable across $\partial \mathbb{D}$ at $\alpha$ and $\left|\psi\left(e^{i \theta}\right)\right|<1$ for $e^{i \theta}$ near to, but not equal to, $\alpha$. More detail can be found in 14, where a somewhat larger class of maps is considered. For such $\alpha$, the curve $\psi\left(e^{i \theta}\right), e^{i \theta}$ near $\alpha$, automatically has positive and even order of contact $2 m$ with $\partial \mathbb{D}$ when $e^{i \theta}=\alpha$; that is,

$$
\frac{1-\left|\psi\left(e^{i \theta}\right)\right|^{2}}{\left|\psi(\alpha)-\psi\left(e^{i \theta}\right)\right|^{2 m}}
$$

is bounded above and away from 0 for $e^{i \theta}$ near $\alpha$. For $k \geq 1$ the $k^{t h}$ order data vector

$$
D_{k}(\psi, \alpha) \equiv\left(\psi(\alpha), \psi^{\prime}(\alpha), \cdots, \psi^{(k)}(\alpha)\right)
$$

makes sense. Given a finite collection $\psi_{1} \cdots, \psi_{n}$ of such maps and $k \geq 2$, we write $\mathcal{M}_{k}(\alpha)$ for the set of integers $j, 1 \leq j \leq n$, for which $F\left(\psi_{j}\right)$ contains $\alpha$ and the order of contact of $\psi_{j}$ at $\alpha$ is at least $k$. Further, put

$$
\mathcal{D}_{k}(\alpha)=\left\{D_{k}\left(\psi_{j}, \alpha\right): j \in \mathcal{M}_{k}(\alpha)\right\},
$$

the set of possible $k^{t h}$ order data vectors at $\alpha$ for associated orders of contact at least $k$. With this notation, Theorem 5.7 in 14 states that for any $k \geq 2$ and complex constants $c_{1} \cdots, c_{n}$,

$$
\left\|c_{1} C_{\psi_{1}}+\cdots+c_{n} C_{\psi_{n}}\right\|_{e}^{2} \geq \sum_{\mathbf{d} \in \mathcal{D}_{k-1}(\alpha)}\left|\sum_{\substack{j \in \mathcal{M}_{k}(\alpha) \\ D_{k-1}\left(\psi_{j}, \alpha\right)=\mathbf{d}}} c_{j}\right|^{2} \frac{1}{\left|d_{1}\right|},
$$

where $\mathbf{d}=\left(d_{0}, d_{1}, \cdots, d_{k-1}\right)$.

For the case $k=2$, we need a calculation which appears in the proof of a more delicate version of the inequality (9); see Lemma 5.9 in 14. Given $\psi$ as above, with $\alpha$ in $F(\psi)$, convert it into a self-map $u$ of the upper half-plane fixing the origin by $u=\tau_{\psi(\alpha)} \circ \psi \circ \tau_{\alpha}^{-1}$. Given our finite collection $\psi_{1} \cdots, \psi_{n}$, associate $u_{j}$ to $\psi_{j}$ in this manner. For $D>0$ we write $\Gamma_{\alpha, D}$ for the locus of the equation $\left(1-|z|^{2}\right) /\left(|\alpha-z|^{2}\right)=4 D$, a circle internally tangent to $\partial \mathbb{D}$ at $\alpha$. We have

$$
\lim _{\Gamma_{\alpha, D}}\left\|\left(\overline{c_{1}} C_{\psi_{1}}^{*}+\cdots+\overline{c_{n}} C_{\psi_{n}}^{*}\right) \frac{k_{z}}{\left\|k_{z}\right\|}\right\|^{2}=\sum_{\mathbf{d} \in \mathcal{D}_{1}(\alpha)}\left\|\sum_{\substack{j \in \mathcal{M}_{2}(\alpha) \\ D_{1}\left(\psi_{j}, \alpha\right)=\mathbf{d}}} \overline{c_{j}} k_{w_{j}}^{+}\right\|_{H_{+}^{2}}^{2}
$$

where $k_{z}$ is the Szego kernel for the Hardy space $H^{2}$ in the disk, $H_{+}^{2}$ is the Hardy space of the right half-plane $\Omega, k_{w}^{+}(z)=1 /(\bar{w}+z)$ is its reproducing kernel, $w_{j}=$ $u_{j}^{\prime}(0) / 2-i D u_{j}^{\prime \prime}(0)$, and the limit is taken as $z \rightarrow \alpha$ along $\Gamma_{\alpha, D}$. Since $u_{j}^{\prime \prime}(0)$ necessarily has non-negative imaginary part, $w_{j}$ is a complex number automatically lying in $\Omega$. For further discussion of this circle of ideas, see [14. We note for future 
reference that a non-automorphism linear-fractional self-map $\psi$ of $\mathbb{D}$ has order of contact two at the unique point in $F(\psi)$.

Finally, we need a variant of a result of Berkson [4] and Shapiro and Sundberg [19], which states that if $\psi_{1}, \cdots, \psi_{n}$ are distinct analytic self-maps of $\mathbb{D}$ and $J\left(\psi_{i}\right)=$ $\left\{e^{i \theta}:\left|\psi_{i}\left(e^{i \theta}\right)\right|=1\right\}$, then for any complex constants $c_{1}, \cdots, c_{n}$,

$$
\left\|c_{1} C_{\psi_{1}}+\cdots+c_{n} C_{\psi_{n}}\right\|_{e}^{2} \geq \frac{1}{2 \pi} \sum_{j=1}^{n}\left|c_{j}\right|^{2}\left|J\left(\psi_{j}\right)\right|,
$$

where $|J|$ denotes the Lebesgue measure of $J$; see Exercise 9.3.2 in [8].

Theorem 1. Let $\psi$ be an analytic self-map of $\mathbb{D}$ such that $C_{\psi}$ lies in $C^{*}\left(C_{\varphi}, \mathcal{K}\right)$, where $\varphi$ is as in (1). If $F(\psi)$ is empty, then $C_{\psi}$ is compact.

Proof. Suppose that $C_{\psi}$ lies in $C^{*}\left(C_{\varphi}, \mathcal{K}\right)$ and $F(\psi)$ is empty. We want to show that $C_{\psi}$ is compact, or equivalently, that the matrix function

$$
\Psi\left(C_{\psi}\right)=\left[\begin{array}{ll}
f_{2} & f_{3} \\
f_{4} & f_{1}
\end{array}\right]
$$

is identically zero on $[0, s]$. Given a small $\epsilon>0$ (size to be specified later), there exists $B$ in $\mathcal{P}$ given by Equation (2) and equivalently by Equation (5), such that $\left\|C_{\psi}-B\right\|<\epsilon$. If we write

$$
Y_{1}=f\left(C_{\varphi}^{*} C_{\varphi}\right), Y_{2}=g\left(C_{\varphi} C_{\varphi}^{*}\right), Y_{3}=C_{\varphi} p\left(C_{\varphi}^{*} C_{\varphi}\right), Y_{4}=C_{\varphi}^{*} q\left(C_{\varphi} C_{\varphi}^{*}\right),
$$

and $Y=Y_{1}+Y_{2}+Y_{3}+Y_{4}$, it is clear that $A_{k} \equiv Y_{k}(\bmod \mathcal{K})$ for each $i$, and $\Psi(Y)=\Psi(A)$, where $A=A_{1}+A_{2}+A_{3}+A_{4}$. Now using the representation (5) for $B$, we have

$$
\left\|C_{\psi}-c C_{z}-A\right\|_{e}<\epsilon
$$

Since $A$ is a finite linear combination of composition operators, we see from the inequality (11) that

$$
\epsilon^{2}>\left\|C_{\psi}-c C_{z}-A\right\|_{e}^{2} \geq \frac{|J(\psi)|}{2 \pi}+|c|^{2} .
$$

From this we find that $|c|<\epsilon$, and since $\epsilon>0$ is arbitrary, $|J(\psi)|=0$. In particular we have $\left\|C_{\psi}-A\right\|_{e}<2 \epsilon$, hence

$$
\left\|\left[\begin{array}{cc}
f_{2}-g & f_{3}-r p \\
f_{4}-r q & f_{1}-f
\end{array}\right]\right\|=\left\|\Psi\left(C_{\psi}-Y\right)\right\|=\left\|C_{\psi}-A\right\|_{e}<2 \epsilon .
$$

It follows that $\left|f_{3}(t)-\sqrt{t} p(t)\right|<2 \epsilon$ for $0 \leq t \leq s$, and similarly for the other three matrix entries.

We will show that $f_{3}$ vanishes identically on $[0, s]$. Suppose not, so that its supremum norm $\left\|f_{3}\right\|_{\infty}$ is positive. Without loss of generality we may assume $8 \epsilon<\left\|f_{3}\right\|_{\infty}$. It follows that there is a non-degenerate closed subinterval $I$ of $[0, s]$, depending only on $f_{3}$ and not containing zero, with $\sqrt{t}|p(t)| \geq\left\|f_{3}\right\|_{\infty} / 2$ for $t$ in $I$. Thus

$$
\int_{I}|p(t)|^{2} d t \geq \frac{\left\|f_{3}\right\|_{\infty}^{2}|I|}{4 s}
$$

We return to this inequality below.

Now we want to apply Equation (10) to the linear combination $A$, which we write as

$$
A=c_{1} C_{\psi_{1}}+\cdots c_{m} C_{\psi_{m}}
$$


Recall that the normalized Szego kernel functions $k_{z} /\left\|k_{z}\right\|$ tend to zero weakly as $|z| \rightarrow 1$, and so

$$
\|T\|_{e}=\left\|T^{*}\right\|_{e} \geq \limsup _{|z| \rightarrow 1}\left\|T^{*}\left(\frac{k_{z}}{\left\|k_{z}\right\|}\right)\right\|
$$

for any bounded operator $T$ on $H^{2}$. The linear-fractional maps $\psi_{1}, \cdots, \psi_{m}$ in Equation (13) are taken from the four lists in (6). The maps in each of these lists have a common angular derivative set (a singleton) and a single common first order data vector. For example, the maps $\psi_{i}$ from the first list all have $F\left(\psi_{i}\right)=\{\eta\}$ and first order data vector $D_{1}\left(\psi_{i}, \eta\right)=(\eta, 1)$, which we call $\mathbf{d}_{1}$. The following table summarizes the corresponding information for each of the four lists:

\begin{tabular}{|c|c|c|}
\hline \multicolumn{3}{|c|}{ TABLE I } \\
\hline \hline$\psi_{i}$ chosen from & $F\left(\psi_{i}\right)$ & $\begin{array}{c}\text { unique first-order } \\
\text { data vector }\end{array}$ \\
\hline$\left\{(\varphi \circ \sigma)_{n_{1}}: n_{1} \geq 1\right\}$ & $\{\eta\}$ & $\mathbf{d}_{1}=(\eta, 1)$ \\
\hline$\left\{(\sigma \circ \varphi)_{n_{2}}: n_{2} \geq 1\right\}$ & $\{\zeta\}$ & $\mathbf{d}_{2}=(\zeta, 1)$ \\
\hline$\left\{(\varphi \circ \sigma)_{n_{3}} \circ \varphi: n_{3} \geq 0\right\}$ & $\{\zeta\}$ & $\mathbf{d}_{3}=\left(\eta, \varphi^{\prime}(\zeta)\right)$ \\
\hline$\left\{(\sigma \circ \varphi)_{n_{4}} \circ \sigma: n_{4} \geq 0\right\}$ & $\{\eta\}$ & $\mathbf{d}_{4}=\left(\zeta, \sigma^{\prime}(\eta)\right)$ \\
\hline
\end{tabular}

Since $C_{\psi}^{*}\left(k_{z}\right)=k_{\psi(z)}$, our hypothesis that $F(\psi)$ is empty says exactly that

$$
\lim _{|z| \rightarrow 1}\left\|C_{\psi}^{*} \frac{k_{z}}{\left\|k_{z}\right\|}\right\|^{2}=\lim _{|z| \rightarrow 1} \frac{1-|z|^{2}}{1-|\psi(z)|^{2}}=0
$$

(see [8, p.132) and thus

$$
\begin{aligned}
4 \epsilon^{2}>\left\|C_{\psi}-A\right\|_{e}^{2} & \geq \limsup _{|z| \rightarrow 1}\left\|\left(C_{\psi}^{*}-A^{*}\right) \frac{k_{z}}{\left\|k_{z}\right\|}\right\|^{2} \\
& \geq \lim _{\Gamma_{\zeta, D}}\left\|\left(\overline{c_{1}} C_{\psi_{1}}^{*}+\cdots+\overline{c_{m}} C_{\psi_{m}}^{*}\right) \frac{k_{z}}{\left\|k_{z}\right\|}\right\|^{2} .
\end{aligned}
$$

We evaluate the limit on the right via Equation (10) with $\alpha=\zeta$. Note that $\mathcal{D}_{1}(\zeta)=\left\{\mathbf{d}_{2}, \mathbf{d}_{3}\right\}$. Discarding the (necessarily non-negative) $\mathbf{d}_{2}$ term from the right-hand side of Equation (10) yields

$$
4 \epsilon^{2}>\lim _{\Gamma_{\zeta, D}}\left\|\left(\overline{c_{1}} C_{\psi_{1}}^{*}+\cdots+\overline{c_{m}} C_{\psi_{m}}^{*}\right) \frac{k_{z}}{\left\|k_{z}\right\|}\right\|^{2} \geq\left\|\sum_{\substack{\zeta \in F\left(\psi_{i}\right) \\ D_{1}\left(\psi_{i}, \zeta\right)=\mathbf{d}_{3}}} \overline{c_{i}} k_{w_{i}}^{+}\right\|_{H_{+}^{2}}^{2} .
$$

We can relabel $\psi_{1}, \cdots, \psi_{m}$ so that the relevant $\psi_{i}^{\prime} s$ occur at the beginning, starting with $i=0$. Then the right-hand side of (14) becomes

$$
\begin{aligned}
\left\|\sum_{k=0}^{n} \overline{c_{k}} k_{w_{k}}^{+}\right\|_{H_{+}^{2}}^{2}=\sum_{i, j=0}^{n} \overline{c_{i}} c_{j} \frac{1}{\overline{w_{i}}+w_{j}} & =\sum_{i, j=0}^{n} \overline{c_{i}} c_{j} \int_{0}^{1} t^{\overline{w_{i}}+w_{j}-1} d t \\
& =\int_{0}^{1}\left|\sum_{k=0}^{n} c_{k} t^{w_{k}}\right|^{2} t^{-1} d t
\end{aligned}
$$


for appropriate $n \leq m-1$.

Let us evaluate $c_{k}$ in terms of the polynomial $p$ occuring in the upper right entry of the matrix function $\Psi(A)=\Psi(Y)$. If

$$
p(z)=\sum_{k=0}^{n} b_{k} z^{k}
$$

we have

$$
\begin{aligned}
Y_{3}=C_{\varphi} p\left(C_{\varphi}^{*} C_{\varphi}\right) & \equiv C_{\varphi} p\left(s C_{\sigma} C_{\varphi}\right)(\bmod \mathcal{K}) \\
& =C_{\varphi} p\left(s C_{\varphi \circ \sigma}\right) \\
& =\sum_{k=0}^{n} b_{k} s^{k} C_{(\varphi \circ \sigma)_{k} \circ \varphi}, \\
& =A_{3},
\end{aligned}
$$

so that, relabeling if necessary, $\psi_{k}=(\varphi \circ \sigma)_{k} \circ \varphi$ and $c_{k}=b_{k} s^{k}$ for $k=0,1, \cdots, n$.

Next we compute $w_{k}$ for $k=0,1, \cdots, n$. Let us convert $\psi_{k}$ into a self-map $U_{k}$ of the upper half-plane fixing the origin as described prior to Equation (10): $U_{k}=$ $\tau_{\eta} \circ \psi_{k} \circ \tau_{\zeta}^{-1}$. We can do the same for the composition factors of $\psi_{k}=(\varphi \circ \sigma)_{k} \circ \varphi$. The map $\varphi \circ \sigma$ is a positive parabolic non-automorphism with fixed point $\eta$. Let $a>0$ be its translation number, so that $\varphi \circ \sigma=\rho_{a}$. Thus for $k \geq 1,(\varphi \circ \sigma)_{k}=\rho_{k a}$, and its half-plane transplant $u_{k}=\tau_{\eta} \circ \rho_{k a} \circ \tau_{\eta}^{-1}$ satisfies $u_{k}^{\prime \prime}(0)=2 i k a$ by Equation (7). We write $v$ for the half-plane version of $\varphi: v=\tau_{\eta} \circ \varphi \circ \tau_{\zeta}^{-1}$. We have

$$
U_{k}=\tau_{\eta} \circ \psi_{k} \circ \tau_{\zeta}^{-1}=\left(\tau_{\eta} \circ \rho_{k a} \circ \tau_{\eta}^{-1}\right) \circ\left(\tau_{\eta} \circ \varphi \circ \tau_{\zeta}^{-1}\right)=u_{k} \circ v .
$$

Now $u_{k}^{\prime}(0)=\rho_{k a}^{\prime}(\eta)=1$ and $v^{\prime}(0)=\left|\varphi^{\prime}(\zeta)\right|=\frac{1}{s}$, and we find

$$
U_{k}^{\prime}(0)=\frac{1}{s} \text { and } U_{k}^{\prime \prime}(0)=v^{\prime \prime}(0)+\frac{2 i k a}{s^{2}} .
$$

From the discussion following Equation (10) we see that

$$
w_{k}=\frac{1}{2 s}-i D v^{\prime \prime}(0)+k\left(\frac{2 a D}{s^{2}}\right) .
$$

To this point $D$ has been an arbitrary positive number. Let us choose $D$ so that $2 a D / s^{2}=1$ and put $\mu=\frac{1}{2 s}-i D v^{\prime \prime}(0)$, a complex number with positive real part. Thus $w_{k}=\mu+k$ and we can write the right hand side of Equation (15) as

$$
\begin{aligned}
\int_{0}^{1}\left|\sum_{k=0}^{n} b_{k} s^{k} t^{k}\right|^{2} t^{2 \operatorname{Re} \mu-1} d t & =\int_{0}^{1}|p(s t)|^{2} t^{2 \operatorname{Re} \mu-1} d t \\
& =\frac{1}{s^{2 \operatorname{Re} \mu}} \int_{0}^{s}|p(t)|^{2} t^{2 \operatorname{Re} \mu-1} d t
\end{aligned}
$$

We consider two cases: if $2 \operatorname{Re} \mu-1 \geq 0$ then this last integral is at least

$$
\frac{t_{0}^{2 \operatorname{Re} \mu-1}}{s^{2 \operatorname{Re} \mu}} \int_{I}|p(t)|^{2} d t
$$

where $t_{0}>0$ is the left-hand endpoint of $I$, and if $2 \operatorname{Re} \mu-1<0$ it is at least

$$
\frac{1}{s} \int_{I}|p(t)|^{2} d t
$$


For small enough $\epsilon>0$, either case of this inequality, combined with the inequality (14) and Equation (15), is incompatible with the inequality (12), yielding the desired contradiction. It follows that $f_{3} \equiv 0$ on $[0, s]$. Entirely similar arguments show that $f_{1}, f_{2}$ and $f_{4}$ vanish identically on $[0, s]$, hence $\Psi\left(C_{\psi}\right)=0$.

With Theorem 1 in hand, we can present our necessary conditions for membership in $C^{*}\left(C_{\varphi}, \mathcal{K}\right)$.

Theorem 2. Let $\varphi$ be as in (1). Suppose $\psi$ is an analytic self-map of $\mathbb{D}$ with $C_{\psi}$ lying in $C^{*}\left(C_{\varphi}, \mathcal{K}\right)$ and $C_{\psi}$ not compact. Then either $\psi(z)=z$ or one of the following holds:

(a) $F(\psi)=\{\zeta\}, \psi(\zeta)=\eta$ and $\psi^{\prime}(\zeta)=\varphi^{\prime}(\zeta)$.

(b) $F(\psi)=\{\zeta\}, \psi(\zeta)=\zeta$ and $\psi^{\prime}(\zeta)=1$.

(c) $F(\psi)=\{\eta\}, \psi(\eta)=\zeta$ and $\psi^{\prime}(\eta)=1 / \varphi^{\prime}(\zeta)$.

(d) $F(\psi)=\{\eta\}, \psi(\eta)=\eta$ and $\psi^{\prime}(\eta)=1$.

(e) $F(\psi)=\{\zeta, \eta\}$ with $\psi(\zeta)=\eta, \psi^{\prime}(\zeta)=\varphi^{\prime}(\zeta), \psi(\eta)=\eta$ and $\psi^{\prime}(\eta)=1$.

(f) $F(\psi)=\{\zeta, \eta\}$ with $\psi(\eta)=\zeta, \psi^{\prime}(\eta)=1 / \varphi^{\prime}(\zeta), \psi(\zeta)=\zeta$ and $\psi^{\prime}(\zeta)=1$.

Proof. If $\psi$ has no finite angular derivative, then Theorem 1 guarantees that $C_{\psi}$ is compact. Thus we may assume $F(\psi)$ is non-empty. We also assume $\psi$ is not the identity, else there is nothing to prove. If $C_{\psi}$ is in $C^{*}\left(C_{\varphi}, \mathcal{K}\right)$, the density of the polynomial subalgebra $\mathcal{P}$ says that given $\epsilon$, we may find a scalar $c$ and a finite linear combination $A$ of composition operators with associated maps from the lists (6) so that

$$
\left\|C_{\psi}-A-c C_{z}\right\|_{e}<\epsilon
$$

As in the beginning of the proof of Theorem 1, we may then conclude that $\left|\psi\left(e^{i \theta}\right)\right|<$ 1 a.e., $|c|<\epsilon$, and that

$$
\left\|C_{\psi}-A\right\|<2 \epsilon
$$

The self-maps of $\mathbb{D}$ which define the composition operators in the linear combination $A$ appear among those in Table I above, along with their angular derivative sets (all singletons) and first order data vectors. Suppose that $\lambda$ is in $F(\psi)$ and $D_{1}(\psi, \lambda)=$ d. If $\lambda$ is not in $\{\zeta, \eta\}$, then the inequality (8) gives

$$
\left\|C_{\psi}-A\right\|_{e}^{2} \geq \frac{1}{\left|\psi^{\prime}(\lambda)\right|}
$$

contradicting (16). Similarly, if $\lambda=\zeta$ and $\mathbf{d}$ is neither $\mathbf{d}_{\mathbf{2}}$ nor $\mathbf{d}_{\mathbf{3}}$ from Table I, or if $\lambda=\eta$ and $\mathbf{d}$ is neither $\mathbf{d}_{\mathbf{1}}$ nor $\mathbf{d}_{\mathbf{4}}$, the inequality (8) and Table I again imply (17). It follows that if $F(\psi)$ is a singleton, one of conditions (a)-(d) must hold.

The remainder of the proof considers the possibility that $F(\psi)=\{\zeta, \eta\}$. The Julia-Caratheodory theory says a non-identity analytic self-map of $\mathbb{D}$ cannot have fixed points at distinct points $\zeta, \eta$ in $\partial \mathbb{D}$ with derivative 1 at each. If we have both $\psi(\zeta)=\eta, \psi^{\prime}(\zeta)=\varphi^{\prime}(\zeta)$ and $\psi(\eta)=\zeta, \psi^{\prime}(\eta)=1 / \varphi^{\prime}(\zeta)$, then $\psi \circ \psi$ fixes both $\zeta$ and $\eta$ with derivative 1 at each, so that $\psi \circ \psi$ is the identity map, contradicting the observation above that $\left|\psi\left(e^{i \theta}\right)\right|<1$ almost everywhere. Thus if $F(\psi)=\{\zeta, \eta\}$, either (e) or (f) must hold, completing the proof.

We will see in Section 5 that there are indeed maps $\psi$ of types (e) and (f) in Theorem 2 for which $C_{\psi}$ belongs to $C^{*}\left(C_{\varphi}, \mathcal{K}\right)$. 


\section{The $C^{*}$-ALGEBRA INDUCED BY PARABOLIC NON-AUTOMORPHISMS}

Let us write $\mathcal{B}\left(H^{2}\right)$ for the algebra of bounded operators on $H^{2}$. A bounded operator $T$ on $H^{2}$ is essentially normal if $T$ and $T^{*}$ commute modulo $\mathcal{K}$; normal operators and compact operators give trivial examples of essentially normal operators. The only normal composition operators $C_{\psi}$ are those of the form $\psi(z)=a z,|a| \leq 1$. Bourdon, Levi, Narayan, and Shapiro 1 showed that if $\psi$ is linear-fractional with $\|\psi\|_{\infty}=1$ and not a rotation, then $C_{\psi}$ is essentially normal exactly when $\psi$ is a parabolic non-automorphism. Let us select a point $\gamma$ in $\partial \mathbb{D}$ and consider the set $\left\{\rho_{a}: \operatorname{Re} a>0\right\}$ of all parabolic non-automorphisms fixing $\gamma$. Here, as earlier, $a$ is the translation number for $\rho_{a}$. Any two of the maps $\rho_{a}$ commute under composition and in fact $\rho_{a} \circ \rho_{b}=\rho_{a+b}$, so $C_{\rho_{a}}$ and $C_{\rho_{b}}$ commute. One can easily check that the Krein adjoint of $\rho_{a}$ is $\rho_{\bar{a}}$. Since $\rho_{a}^{\prime}(\gamma)=1$, it follows from Equation (4) that $C_{\rho_{a}}^{*}=C_{\rho_{\bar{a}}}+K$ for some compact operator $K$. A recent theorem of Montes-Rodríguez, Ponce-Escudero and Shkarin [18] shows that $C_{\rho_{a}}$ is irreducible. Moreover $C^{*}\left(C_{\rho_{a}}\right)$, the unital $C^{*}$-algebra generated by $C_{\rho_{a}}$, contains the commutator of $C_{\rho_{a}}$ and $C_{\rho_{a}}^{*}$ which we know is compact but non-zero. Thus $C^{*}\left(C_{\rho_{a}}\right)$ contains $\mathcal{K}$ and $C^{*}\left(C_{\rho_{a}}\right) / \mathcal{K}$ is commutative. Now let $\mathbb{P}_{\gamma}$ denote the set of all composition operators $C_{\rho}$, where $\rho$, fixing $\gamma$, ranges over $\left\{\rho_{a}: \operatorname{Re} a>0\right\}$. We write $C^{*}\left(\mathbb{P}_{\gamma}\right)$ for the unital $C^{*}$-algebra generated by the operators in $\mathbb{P}_{\gamma}$. Clearly $C^{*}\left(\mathbb{P}_{\gamma}\right)$ contains $\mathcal{K}$, and, by the above remarks, $C^{*}\left(\mathbb{P}_{\gamma}\right) / \mathcal{K}$ is also commutative. In this section we compute and apply the Gelfand representation of this quotient algebra.

We begin with two lemmas.

Lemma 1. For $a>0$ there is an operator $A \geq 0$ and a compact operator $K$ with $C_{\rho_{a}}=A+K$.

Proof. Then

$$
\begin{aligned}
C_{\rho_{a / 2}} & =\frac{1}{2}\left(C_{\rho_{a / 2}}+C_{\rho_{a / 2}}^{*}\right)+\frac{1}{2}\left(C_{\rho_{a / 2}}-C_{\rho_{a / 2}}^{*}\right) \\
& \equiv B+J
\end{aligned}
$$

where $B$ is self-adjoint and $J$ is compact. Thus $C_{\rho_{a}}=C_{\rho_{a / 2}} C_{\rho_{a / 2}}=(B+J)^{2}=$ $B^{2}+\left(B J+J B+J^{2}\right)$. Since $B^{2}$ is positive and $B J+J B+J^{2}$ is compact, we are done.

Lemma 2. Let $a, b$ be positive with $b / a=m / n$, with $m$ and $n$ positive integers. Suppose $0<\lambda \leq 1$ and there is a sequence $f_{k}$ of unit vectors in $H^{2}$ converging weakly to zero such that

$$
\left\|\left(C_{\rho_{a}}-\lambda\right) f_{k}\right\| \rightarrow 0
$$

Then

$$
\left\|\left(C_{\rho_{b}}-\lambda^{m / n}\right) f_{k}\right\| \rightarrow 0
$$

Proof. First observe that

$$
C_{\rho_{a}}^{m}-\lambda^{m}=\left[C_{\rho_{a}}^{m-1}+\lambda C_{\rho_{a}}^{m-2}+\cdots+\lambda^{m-2} C_{\rho_{a}}+\lambda^{m-1}\right]\left[C_{\rho_{a}}-\lambda\right] .
$$

In particular, $\left\|\left(C_{\rho_{a}}^{m}-\lambda^{m}\right) f_{k}\right\| \rightarrow 0$ as $k \rightarrow \infty$. Since $C_{\rho_{a}}^{m}=C_{\rho_{b}}^{n}$,

$$
\left\|\left(C_{\rho_{b}}^{n}-\lambda^{m}\right) f_{k}\right\| \rightarrow 0 .
$$

Also note that we may factor $C_{\rho_{b}}^{n}-\lambda^{m}=C_{\rho_{b}}^{n}-\left(\lambda^{m / n}\right)^{n}$ as

$$
\left[C_{\rho_{b}}^{n-1}+\lambda^{m / n} C_{\rho_{b}}^{n-2}+\cdots+\left(\lambda^{m / n}\right)^{n-2} C_{\rho_{b}}+\left(\lambda^{m / n}\right)^{n-1}\right]\left[C_{\rho_{b}}-\lambda^{m / n}\right] .
$$


Apply Lemma 1 to $C_{\rho_{b}}$ to write

$$
C_{\rho_{b}}^{n-1}+\lambda^{m / n} C_{\rho_{b}}^{n-2}+\cdots+\left(\lambda^{m / n}\right)^{n-2} C_{\rho_{b}}+\left(\lambda^{m / n}\right)^{n-1} I=T+\left(\lambda^{m / n}\right)^{n-1} I+K
$$

for some positive $T$ and compact $K$. We have

$$
\left\|\left(C_{\rho_{b}}^{n}-\lambda^{m}\right) f_{k}\right\|=\left\|\left(T+\left(\lambda^{m / n}\right)^{n-1}+K\right)\left(C_{\rho_{b}}-\lambda^{m / n}\right) f_{k}\right\| .
$$

Since $K$ is compact, $\left\|K\left(C_{\rho_{b}}-\lambda^{m / n}\right) f_{k}\right\| \rightarrow 0$ as $k \rightarrow \infty$. Since the left-hand side of Equation (18) goes to 0 as $k \rightarrow \infty$, we see, writing $c=\left(\lambda^{m / n}\right)^{n-1}$ that

$$
\left\|(T+c I)\left(C_{\rho_{b}}-\lambda^{m / n}\right) f_{k}\right\| \rightarrow 0 .
$$

But $\left\|(T+c I)\left(C_{\rho_{b}}-\lambda^{m / n}\right) f_{k}\right\|^{2}$ is equal to

$$
\begin{aligned}
\left\|T\left(C_{\rho_{b}}-\lambda^{m / n}\right) f_{k}\right\|^{2} & +2 c\left\langle T\left(C_{\rho_{b}}-\lambda^{m / n}\right) f_{k},\left(C_{\rho_{b}}-\lambda^{m / n}\right) f_{k}\right\rangle \\
& +c^{2}\left\|\left(C_{\rho_{b}}-\lambda^{m / n}\right) f_{k}\right\|^{2} \geq c^{2}\left\|\left(C_{\rho_{b}}-\lambda^{m / n}\right) f_{k}\right\|^{2}
\end{aligned}
$$

where the last inequality uses the positivity of $T$, so that $\left\|\left(C_{\rho_{b}}-\lambda^{m / n}\right) f_{k}\right\| \rightarrow 0$.

The essential spectrum $\sigma_{e}(T)$ of a bounded operator on $H^{2}$ is by definition the spectrum of the coset $[T]$ in $\mathcal{B}\left(H^{2}\right) / \mathcal{K}$. We recall from [15] that if $a>0, \sigma_{e}\left(C_{\rho_{a}}\right)=$ $[0,1]$. We will need the notion of joint essential spectrum, which is treated by Dash in [9]. If $\operatorname{Re} a>0$, the coset $\left[C_{\rho_{a}}\right]$ of $C_{\rho_{a}}$ modulo $\mathcal{K}$ will also be denoted by $x_{a}$. By either Lemma 1 or the discussion preceeding it, $x_{a}$ is self-adjoint. Given $a$ and $b$, the joint essential spectrum $\sigma_{e}\left(C_{\rho_{a}}, C_{\rho_{b}}\right)$ is defined to be the joint spectrum $\sigma\left(x_{a}, x_{b}\right)$ of the pair $x_{a}, x_{b}$ in the Calkin algebra $\mathcal{B}\left(H^{2}\right) / \mathcal{K}$. This set coincides with the joint spectrum in the commutative unital subalgebra $C^{*}\left(x_{a}, x_{b}\right)$ generated by $x_{a}$ and $x_{b}$. If $\mathcal{M}$ is the maximal ideal space of this algebra, and ${ }^{-}$denotes the Gelfand transform, then the map $\ell \mapsto\left(\widehat{x_{a}}(\ell), \widehat{x_{b}}(\ell)\right)$ is a homeomorphism of $\mathcal{M}$ onto $\sigma\left(x_{a}, x_{b}\right)$. Let us assume that $a$ and $b$ are positive. A theorem of Dash 9 ] states, in this context, using $C_{\rho_{a}}^{*} \equiv C_{\rho_{a}}(\bmod \mathcal{K})$ and similarly for $C_{\rho_{b}}$, that $(\lambda, \mu)$ lies in $\sigma_{e}\left(C_{\rho_{a}}, C_{\rho_{b}}\right)$ if and only if there exists a sequence $\left\{f_{k}\right\}$ of unit vectors in $H^{2}$, converging weakly to zero, such that $\left\|\left(C_{\rho_{a}}-\lambda\right) f_{k}\right\|$ and $\left\|\left(C_{\rho_{b}}-\mu\right) f_{k}\right\|$ both tend to zero as $k \rightarrow \infty$.

Corollary 1. Suppose that $a, b$ are positive and $b / a$ is rational. Then

$$
\sigma_{e}\left(C_{\rho_{a}}, C_{\rho_{b}}\right)=\left\{\left(t^{a}, t^{b}\right): 0 \leq t \leq 1\right\} .
$$

Proof. We know that

$$
\sigma_{e}\left(C_{\rho_{a}}, C_{\rho_{b}}\right) \subset \sigma_{e}\left(C_{\rho_{a}}\right) \times \sigma_{e}\left(C_{\rho_{b}}\right)=[0,1] \times[0,1] .
$$

Let $0<\lambda \leq 1$. Since $\lambda \in \sigma_{e}\left(C_{\rho_{a}}\right)$, we may find unit vectors $f_{k}$ with $f_{k} \rightarrow 0$ weakly and $\left\|\left(C_{\rho_{a}}-\lambda\right) f_{k}\right\| \rightarrow 0$. By Lemma 2, $\left\|\left(C_{\rho_{b}}-\lambda^{b / a}\right) f_{k}\right\| \rightarrow 0$ as well, so that $\left(\lambda, \lambda^{b / a}\right)$ is in $\sigma_{e}\left(C_{\rho_{a}}, C_{\rho_{b}}\right)$ by Dash's theorem. Setting $t^{a}=\lambda$ we have $\left\{\left(t^{a}, t^{b}\right): 0<t \leq 1\right\} \subset \sigma_{e}\left(C_{\rho_{a}}, C_{\rho_{b}}\right)$. The set on the right is compact in $\mathbb{R}^{2}$, so it contains $(0,0)$ as well, giving $\left\{\left(t^{a}, t^{b}\right): 0 \leq t \leq 1\right\} \subset \sigma_{e}\left(C_{\rho_{a}}, C_{\rho_{b}}\right)$. Conversely, if $(\lambda, \mu) \in \sigma_{e}\left(C_{\rho_{a}}, C_{\rho_{b}}\right)$, Dash's theorem gives the existence of a sequence of unit vectors $f_{k}$ converging weakly to 0 with both $\left\|\left(C_{\rho_{a}}-\lambda\right) f_{k}\right\|$ and $\left\|\left(C_{\rho_{b}}-\mu\right) f_{k}\right\|$ tending to zero as $k \rightarrow \infty$. If $\lambda>0,\left\|\left(C_{\rho_{b}}-\lambda^{b / a}\right) f_{k}\right\| \rightarrow 0$ by Lemma 2. Thus $\mu=\lambda^{b / a}$ and $(\lambda, \mu)=\left(\lambda, \lambda^{b / a}\right)=\left(t^{a}, t^{b}\right)$ for some $t, 0 \leq t \leq 1$. If $\mu>0$, the symmetric result says $\lambda=\mu^{a / b}>0$, and, putting $\mu=t^{b},(\lambda, \mu)=\left(\mu^{a / b}, \mu\right)=\left(t^{a}, t^{b}\right)$, again of the desired form. As for $(0,0)$, we already know it lies in $\sigma_{e}\left(C_{\rho_{a}}, C_{\rho_{b}}\right)$, and of course it has the form $\left(0^{a}, 0^{b}\right)$. 
We will need the fact that on the domain $\Omega=\{a: \operatorname{Re} a>0\}$ the map $a \mapsto C_{\rho_{a}}$ is a holomorphic function of $a$ in the operator norm topology; see for example the discussion in the proof of Theorem 6.1 in [6]. We continue to denote the coset of $C_{\rho_{a}}$ by $x_{a}$ and to keep in mind that when $a>0, \sigma\left(x_{a}\right)=[0,1]$.

Theorem 3. There is a unique $*$-isomorphism $\Gamma: C([0,1]) \rightarrow C^{*}\left(\mathbb{P}_{\gamma}\right) / \mathcal{K}$ such that $\Gamma\left(t^{a}\right)=\left[C_{\rho_{a}}\right]$ for all $a \in \Omega$.

Proof. First consider $a=1$ and $x_{1}=\left[C_{\rho_{1}}\right]$. Since $\sigma\left(x_{1}\right)=[0,1]$ we may define a *-isomorphism $\Gamma: C([0,1]) \mapsto C^{*}\left(x_{1}\right)$ by sending $p$ to $p\left(x_{1}\right)$ for any polynomial $p$. Fix any rational number $r>0$. By Corollary 1 ,

$$
\sigma\left(x_{1}, x_{r}\right)=\sigma_{e}\left(C_{\rho_{1}}, C_{\rho_{r}}\right)=\left\{\left(t, t^{r}\right): 0 \leq t \leq 1\right\} .
$$

The map $p\left(x_{1}, x_{r}\right) \mapsto p\left(z_{1}, z_{2}\right)$, where $p$ is a two-variable polynomial, extends to a unique *-isomorphism of $C^{*}\left(x_{1}, x_{r}\right)$ onto $C\left(\sigma\left(x_{1}, x_{r}\right)\right)$. Since $\sigma\left(x_{1}, x_{r}\right)$ is homeomorphic to $[0,1]$ via the map $t \mapsto\left(t, t^{r}\right)$, we see that $p\left(x_{1}, x_{r}\right) \mapsto p\left(t, t^{r}\right)$ defines a *-isomorphism of $C^{*}\left(x_{1}, x_{r}\right)$ onto $C([0,1])$. Let $\tilde{\Gamma}$ denote the inverse of this map, that is $\tilde{\Gamma}: p\left(t, t^{r}\right) \mapsto p\left(x_{1}, x_{r}\right)$. Since polynomials in $t$ span $C([0,1]), x_{1}$ generates the $C^{*}$-algebra $C^{*}\left(x_{1}, x_{r}\right)$ and $C^{*}\left(x_{1}\right)=C^{*}\left(x_{1}, x_{r}\right)$. It follows that $\tilde{\Gamma}=\Gamma$. Since $r$ is arbitrary in the set $\mathbb{Q}_{+}$of positive rationals, we have shown that

$$
C^{*}\left(\left\{x_{r}: r \in \mathbb{Q}_{+}\right\}\right)=C^{*}\left(x_{1}\right) .
$$

Moreover, $\Gamma\left(t^{r}\right)=x_{r}, r \in \mathbb{Q}_{+}$. It is easy to see that the map $a \mapsto t^{a}$ is a normholomorphic map of the right half plane into $C([0,1])$ and thus that $a \mapsto \Gamma\left(t^{a}\right)$ is norm- holomorphic from the right half-lane to $\mathcal{B}\left(H^{2}\right) / \mathcal{K}$, as is the function $a \mapsto x_{a}$. We have seen that these functions agree on $\mathbb{Q}_{+}$, hence they must agree on the right half-plane $\Omega$.

We record three immediate consequences.

Corollary 2. If $a_{1}, \cdots, a_{n}$ lie in the right half-plane $\Omega$, then

$$
\sigma_{e}\left(C_{\rho_{a_{1}}}, \cdots, C_{\rho_{a_{n}}}\right)=\left\{\left(t^{a_{1}}, \cdots, t^{a_{n}}\right): 0 \leq t \leq 1\right\} .
$$

Corollary 3. If $\rho$ is a parabolic non-automorphism fixing $\gamma$, then $C^{*}\left(C_{\rho}\right)=$ $C^{*}\left(\mathbb{P}_{\gamma}\right)$.

Corollary 4. If $\varphi$ is as in (1), then $\mathbb{P}_{\zeta}$ and $\mathbb{P}_{\eta}$ are both subsets of $C^{*}\left(C_{\varphi}, \mathcal{K}\right)$.

\section{LINEAR-FRACTIONAL MAPS}

The goal of this section is to find all linear-fractional $\psi$ with $C_{\psi}$ in $C^{*}\left(C_{\varphi}, \mathcal{K}\right)$, where $\varphi$ satisfies the conditions of (1). Since $C_{\psi}$ is compact if $\|\psi\|_{\infty}<1$, our interest is in the case $\|\psi\|_{\infty}=1$.

Lemma 3. If $\varphi$ is as in (1), $C^{*}\left(C_{\varphi}, \mathcal{K}\right)$ contains $C_{\psi}$ for all linear-fractional $\psi$ : $\mathbb{D} \rightarrow \mathbb{D}$ with $\psi(\zeta)=\eta, \psi^{\prime}(\zeta)=\varphi^{\prime}(\zeta)$ and $\psi(\mathbb{D})$ properly contained in $\varphi(\mathbb{D})$.

Proof. Set $\tau=\varphi^{-1} \circ \psi$, noting that the hypothesis $\psi(D) \subset \varphi(\mathbb{D})$ means that $\tau$ is well-defined. Since this containment is proper, and $\tau^{\prime}(\zeta)=1, \tau$ is a parabolic nonautomorphism with fixed point $\zeta$. Since $\varphi \circ \tau=\psi, C_{\psi}=C_{\tau} C_{\varphi}$. By Corollary 4 $C_{\tau} \in C^{*}\left(C_{\varphi}, \mathcal{K}\right)$, from which the conclusion follows. 
Now consider the parabolic non-automorphism $\rho=\varphi \circ \sigma$. The unique fixed point for $\rho$ and its iterates $(\rho)_{n}$ is $\eta$. Fix an integer $n \geq 1$ and let $\varphi_{1}=(\rho)_{n} \circ \varphi$. Clearly $\varphi_{1}(\mathbb{D})$ is properly contained in $\varphi(\mathbb{D})$. Note that $\varphi_{1}(\zeta)=\eta$ and, since $\rho^{\prime}(\eta)=1, \varphi_{1}^{\prime}(\zeta)=\varphi^{\prime}(\zeta)$. It follows from Lemma 3 that $C^{*}\left(C_{\varphi_{1}}, \mathcal{K}\right)$ is contained in $C^{*}\left(C_{\varphi}, \mathcal{K}\right)$. Let $i$ denote the corresponding inclusion map. Since $i(\mathcal{K})=\mathcal{K}, i$ induces a $*$-homomorphism

$$
\hat{i}: C^{*}\left(C_{\varphi_{1}}, \mathcal{K}\right) / \mathcal{K} \rightarrow C^{*}\left(C_{\varphi}, \mathcal{K}\right) / \mathcal{K}
$$

given by $\hat{i}([T])=[T]$, where $[T]$ denotes the coset, modulo $\mathcal{K}$ of the operator $T$. Note that $\hat{i}$ is itself an inclusion. Also observe that the map $\Psi: C^{*}\left(C_{\varphi}, \mathcal{K}\right) \rightarrow \mathcal{D}$ induces a $*$-isomorphism $\Phi: C^{*}\left(C_{\varphi}, \mathcal{K}\right) / \mathcal{K} \rightarrow \mathcal{D}$ given by $\Phi([T])=\Psi(T)$. Let $\Phi_{1}$ denote the corresponding $*$-isomorphism $\Phi_{1}: C^{*}\left(C_{\varphi_{1}}, \mathcal{K}\right) / \mathcal{K} \rightarrow \mathcal{D}$. Keep in mind that $\Phi_{1}$ should be defined by $\Phi_{1}([T])=\Psi_{1}(T)$, where $\Psi_{1}: C^{*}\left(C_{\varphi_{1}}, \mathcal{K}\right) \rightarrow \mathcal{D}$ is associated to $\varphi_{1}$ as $\Psi$ is associated to $\varphi$. Thus if $B$ in $C^{*}\left(C_{\varphi_{1}}, \mathcal{K}\right)$ is given by (2), but with $\varphi$ replaced by $\varphi_{1}$, then $\Psi_{1}(B)$ is given by (3). We have a commutative diagram

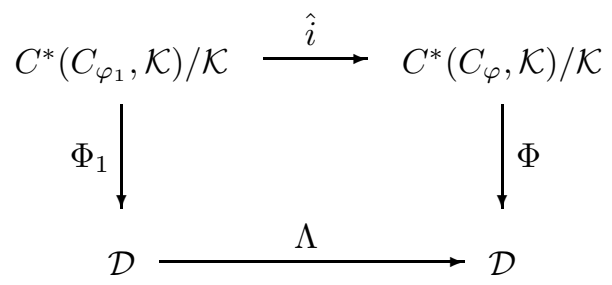

where $\Lambda=\Phi \circ \hat{i} \circ \Phi_{1}^{-1}$. We seek to identify $\Lambda$ explicitly.

Lemma 4. For any element $F$ in $\mathcal{D}$,

$$
(\Lambda F)(t)=F\left(t^{2 n+1} / s^{2 n}\right), 0 \leq t \leq s
$$

Proof. For the purposes of the proof, we use $\Lambda$ to denote the map given by formula (19), and then show, with this redefinition, that it coincides with $\Phi \circ \hat{i} \circ \Phi_{1}^{-1}$, that is, that $\Lambda \circ \Phi_{1}=\Phi \circ \hat{i}$. Recall that $C_{\varphi}^{*} \equiv s C_{\sigma}(\bmod \mathcal{K})$ so that

$$
\begin{aligned}
C_{\varphi_{1}}=C_{\varphi} C_{(\rho)_{n}} & =C_{\varphi}\left(C_{\sigma} C_{\varphi}\right)^{n} \\
& \equiv \frac{1}{s^{n}} C_{\varphi}\left(C_{\varphi}^{*} C_{\varphi}\right)^{n}(\bmod \mathcal{K}),
\end{aligned}
$$

and, taking adjoints, $C_{\varphi_{1}}^{*} \equiv \frac{1}{s^{n}} C_{\varphi}^{*}\left(C_{\varphi} C_{\varphi}^{*}\right)^{n}$ modulo the compacts. Calculations using these two facts show that if we write $y=\left[C_{\varphi_{1}}\right]$ and $x=\left[C_{\varphi}\right]$, we have, for each non-negative integer $m$,

$$
\begin{gathered}
\left(y^{*} y\right)^{m}=\frac{1}{s^{2 n m}}\left(x^{*} x\right)^{(2 n+1) m}, \\
\left(y y^{*}\right)^{m}=\frac{1}{s^{2 n m}}\left(x x^{*}\right)^{(2 n+1) m} . \\
y\left(y^{*} y\right)^{m}=\frac{1}{s^{(2 m+1) n}} x\left(x^{*} x\right)^{(2 n+1) m+n},
\end{gathered}
$$

and

$$
y^{*}\left(y y^{*}\right)^{m}=\frac{1}{s^{(2 m+1) n}} x^{*}\left(x x^{*}\right)^{(2 n+1) m+n} .
$$


The left-hand sides in these four equations are elements in $C^{*}\left(C_{\varphi_{1}}, \mathcal{K}\right) / \mathcal{K}$, while the right-hand sides represent the same objects as elements of $C^{*}\left(C_{\varphi}, \mathcal{K}\right) / \mathcal{K}$. We first act on $y\left(y^{*} y\right)^{m}$ by $\hat{i}$, followed by $\Phi$. We then act on $y\left(y^{*} y\right)^{m}$ by $\Phi_{1}$, followed by $\Lambda$ (as defined by Equation (19)). As the reader can see from the following picture, we end up with a common result, the matrix function in the lower right-hand corner.

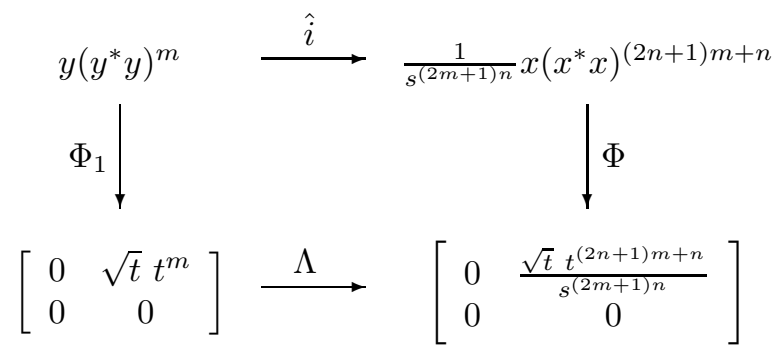

One can check that we also arrive at common values when $\Lambda \circ \Phi_{1}$ and $\Phi \circ \hat{i}$ act on $\left(y^{*} y\right)^{m}$, and similarly for $\left(y y^{*}\right)^{m}$ and $y^{*}\left(y y^{*}\right)^{m}$. Since elements of the form $\left(y^{*} y\right)^{m}$, $\left(y y^{*}\right)^{m}, y\left(y^{*} y\right)^{m}$ and $y^{*}\left(y y^{*}\right)^{m}$, together with the identity, span $C^{*}\left(C_{\varphi_{1}}, \mathcal{K}\right) / \mathcal{K}$, we have $\Lambda \circ \Phi_{1}=\Phi \circ \hat{i}$ as desired

It is clear from (19) that $\Lambda$ is an automorphism of $\mathcal{D}$. It follows that $\hat{i}$ is an isomorphism and thus that $i$ has range equal to all of $C^{*}\left(C_{\varphi}, \mathcal{K}\right)$, that is,

$$
C^{*}\left(C_{\varphi_{1}}, \mathcal{K}\right)=C^{*}\left(C_{\varphi}, \mathcal{K}\right)
$$

More generally, we have the following result.

Theorem 4. Let $\psi$ be a linear-fractional map of $\mathbb{D}$, not an automorphism, with $\psi(\zeta)=\varphi(\zeta)$ and $\psi^{\prime}(\zeta)=\varphi^{\prime}(\zeta)$, where $\varphi$ is as in (11). Then $C^{*}\left(C_{\psi}, \mathcal{K}\right)=C^{*}\left(C_{\varphi}, \mathcal{K}\right)$.

Proof. The circles $\varphi(\partial \mathbb{D})$ and $\psi(\partial \mathbb{D})$ are both internally tangent to $\partial \mathbb{D}$ at $\eta$. If $\varphi(\mathbb{D})$ is a proper subset of $\psi(\mathbb{D})$, then

$$
C^{*}\left(C_{\varphi}, \mathcal{K}\right) \subset C^{*}\left(C_{\psi}, \mathcal{K}\right)
$$

by Lemma 3. Suppose on the other hand that $\psi(\mathbb{D}) \subset \varphi(\mathbb{D})$. If $a$ is the (necessarily positive) translation number of the parabolic map $\varphi \circ \sigma$ (so that $\varphi \circ \sigma=\rho_{a}$ in the terminology of Section 2), then $(\varphi \circ \sigma)_{n}=\rho_{n a}$, and the radius of the disk $\rho_{n a}(\mathbb{D})$ shrinks to zero as $n \rightarrow \infty$. Thus there exists $n$ with $\rho_{n a}(\mathbb{D})$ properly contained in $\psi(D)$. If $\varphi_{1}=(\varphi \circ \sigma)_{n} \circ \varphi=\rho_{n a} \circ \varphi$, then $\varphi_{1}(\mathbb{D})$ is also properly contained in $\psi(\mathbb{D})$. Since $\varphi_{1}(\zeta)=\eta=\psi(\zeta)$ and $\varphi_{1}^{\prime}(\zeta)=\rho_{n a}^{\prime}(\eta) \varphi^{\prime}(\zeta)=\varphi^{\prime}(\zeta)=\psi^{\prime}(\zeta)$, Lemma 3 implies that $C^{*}\left(C_{\psi}, \mathcal{K}\right)$ contains $C^{*}\left(C_{\varphi_{1}}, \mathcal{K}\right)$, which by (20) coincides with $C^{*}\left(C_{\varphi}, \mathcal{K}\right)$. The result is that (21) holds, whatever the relationship between the disks $\varphi(\mathbb{D})$ and $\psi(\mathbb{D})$. The statement of the theorem is symmetric in $\varphi$ and $\psi$, so symmetry implies that the containment reverse to that in (21) also holds, completing the proof.

Theorem 5. Suppose $\varphi$ is as in (1). Let $\psi$, not the identity, be any linear-fractional self-map of $\mathbb{D}$ with $\|\psi\|_{\infty}=1$. Then $C_{\psi}$ is in $C^{*}\left(C_{\varphi}, \mathcal{K}\right)$ if and only if $\psi$ is not an automorphism and one of the following conditions holds:

(a) $\psi(\zeta)=\eta$ and $\psi^{\prime}(\zeta)=\varphi^{\prime}(\zeta)$.

(b) $\psi(\zeta)=\zeta$ and $\psi^{\prime}(\zeta)=1$.

(c) $\psi(\eta)=\zeta$ and $\psi^{\prime}(\eta)=1 / \varphi^{\prime}(\zeta)$.

(d) $\psi(\eta)=\eta$ and $\psi^{\prime}(\eta)=1$. 
Proof. The "only if" statement follows immediately from Theorem 2 and the hypothesis that $\psi$ is linear-fractional. Conversely, let $\psi$ be a linear-fractional map which is not an automorphism. If $\psi$ is parabolic with fixed point at either $\zeta$ or $\eta$, the result follows from Corollary 4 this handles the cases (b) and (d). If $\psi$ is as in (a), then we have $C_{\psi} \in C^{*}\left(C_{\varphi}, \mathcal{K}\right)$ by Theorem 4 Finally, if $\psi$ satisfies condition (c), then its Krein adjoint $\sigma_{\psi}$ is a linear-fractional self-map of $\mathbb{D}$, not an automorphism, which satisfies condition (a), so that $C_{\sigma_{\psi}} \in C^{*}\left(C_{\varphi}, \mathcal{K}\right)$. Since $C_{\psi}^{*} \equiv s C_{\sigma_{\psi}}$ modulo the compacts, this completes the argument.

The maps satisfying (a)-(d) in Theorem 5 can be described more explicitly. Given a point $\gamma$ on $\partial \mathbb{D}$, let us write $\rho_{\gamma, a}$ for the unique parabolic map fixing $\gamma$ with translation number $a$. This will be a self-map of $\mathbb{D}$ when $\operatorname{Re} a \geq 0$, but when $\operatorname{Re} a<0, \rho_{\gamma, a}$ takes $\mathbb{D}$ onto a larger disk, whose boundary is externally tangent to $\partial \mathbb{D}$ at $\gamma$. Clearly, the linear-fractional non-automorphisms of $\mathbb{D}$ satisfying (b) or (d) are, respectively, $\rho_{\zeta, a}$ or $\rho_{\eta, a}$ with Re $a>0$. Imaginary $a$ gives an automorphism of $\mathbb{D}$, but in this case $C^{*}\left(C_{\varphi}, \mathcal{K}\right)$ does not contain the corresponding composition operator.

Consider now a linear-fractional non-automorphism $\psi$ of $\mathbb{D}$ satisfying (a). If $\psi(\mathbb{D}) \subset \varphi(\mathbb{D})$, we can define $\rho=\psi \circ \varphi^{-1}$ which fixes $\eta$ and carries $\mathbb{D}$ to $\mathbb{D}$. Moreover, $\rho^{\prime}(\eta)=\psi^{\prime}(\zeta) / \varphi^{\prime}(\zeta)=1$, so $\rho$ is parabolic; say $\rho=\rho_{\eta, a}$ where Re $a \geq 0$, and we find $\psi=\rho_{\eta, a} \circ \varphi$. On the other hand, if $\psi$ satisfies (a) and $\varphi(\mathbb{D})$ is a proper subset of $\psi(\mathbb{D})$, we put $\rho=\varphi \circ \psi^{-1}$ which is again a parabolic self-map of $\mathbb{D}$; this time a nonautomorphism. Thus $\rho=\rho_{\eta, a}$ with $\operatorname{Re} a>0$ and we find $\psi=\rho_{\eta, a}^{-1} \circ \varphi=\rho_{\eta,-a} \circ \varphi$. If $b$ is the unique positive number with $\rho_{\eta, b}(\mathbb{D})=\varphi(\mathbb{D})$, then $\rho_{\eta,-a} \circ \varphi$ is a nonautomorphism self-map of $\mathbb{D}$ exactly when Re $a<b$. Rephrasing and summarizing, we conclude that the non-automorphisms $\psi$ of $\mathbb{D}$ satisfying (a) are precisely the maps of the form $\rho_{\eta, a} \circ \varphi$, Re $a>-b$. Similarly, if $c$ is the unique positive number with $\rho_{\zeta, c}(\mathbb{D})=\sigma(\mathbb{D})$, then the non-automorphisms $\psi$ satisfying (c) are exactly the maps of the form $\psi=\rho_{\zeta, a} \circ \sigma$ with $\operatorname{Re} a>-c$. The next result shows that the positive translation numbers $b$ and $c$ are nicely related to each other, and to the translation numbers of the positive parabolic non-automorphisms $\varphi \circ \sigma$ and $\sigma \circ \varphi$.

Theorem 6. Let $b$ and $c$ be the unique positive numbers with $\rho_{\eta, b}(\mathbb{D})=\varphi(\mathbb{D})$ and $\rho_{\zeta, c}(\mathbb{D})=\sigma(\mathbb{D})$, respectively. We have $c=\left|\varphi^{\prime}(\zeta)\right| b$, and moreover, $\varphi \circ \sigma=\rho_{\eta, 2 b}$ and $\sigma \circ \varphi=\rho_{\zeta, 2 c}$.

Proof. Clearly there is no loss of generality in assuming that $\zeta=1$. The non-affine linear-fractional self-maps of $\mathbb{D}$ which send 1 to $\eta \in \partial \mathbb{D}$ can be written in the form

$$
\varphi(z)=\eta \frac{(1+s+s d) z+(d-s-s d)}{z+d}
$$

where $s=\left|\varphi^{\prime}(1)\right|$ and $\operatorname{Re} \frac{d-1}{d+1} \geq s$ (see [3]). A computation shows that $\varphi^{\prime}(1)=\eta s$ and $\varphi^{\prime \prime}(1)=-2 \eta s /(1+d)$. The image of the unit circle under $\varphi$ is a circle with curvature $\kappa_{1}=\left|\varphi^{\prime}(1)\right|^{-1} \operatorname{Re}\left[1+\varphi^{\prime \prime}(1) / \varphi^{\prime}(1)\right]=\frac{1}{s} \operatorname{Re}\left[1-\frac{2}{1+d}\right]$. Since

$$
\sigma(z)=\frac{\bar{\eta}(1+s+s \bar{d}) z-1}{-\bar{\eta}(\bar{d}-s-s \bar{d}) z+\bar{d}},
$$

we find that $\sigma^{\prime}(\eta)=\bar{\eta} / s$ and

$$
\sigma^{\prime \prime}(\eta)=\frac{2 \bar{\eta}^{2}(\bar{d}-s-s \bar{d})}{s^{2}(1+\bar{d})},
$$


so that the image of the unit circle under $\sigma$ is a circle with curvature

$$
\kappa_{2}=s \operatorname{Re}\left\{1+\eta \frac{2 \bar{\eta}^{2}(\bar{d}-s-s \bar{d})}{s^{2}(1+\bar{d})} \cdot \frac{s}{\bar{\eta}}\right\}=2-2 \operatorname{Re} \frac{1}{1+d}-s .
$$

A positive parabolic non-automorphism fixing 1 and corresponding to translation by $a$ has the form $((2+d) z-1) /(z+d)$ where $a=-2 /(d+1)$; by the above calculations the image of the unit circle under this map has curvature $1+\operatorname{Re} a$. Thus, if $\varphi(1)=\eta$ and $\left|\varphi^{\prime}(1)\right|=s$, the unique positive value $b$ such that the curvature of $\varphi(\partial \mathbb{D})$ is equal to the curvature of the image of $\partial \mathbb{D}$ under the positive parabolic map which corresponds to translation by $b>0$ satisfies

$$
1+b=\frac{1}{s} \operatorname{Re}\left(1-\frac{2}{1+d}\right) ;
$$

that is,

$$
b=\frac{1}{s} \operatorname{Re}\left(1-\frac{2}{1+d}\right)-1
$$

Similarly, the curvature of the circle $\sigma(\partial \mathbb{D})$ is equal to the curvature of the circle which is the image of the unit circle under the positive parabolic non-automorphism corresponding to translation by $c$ precisely when $c=1-s-2 \operatorname{Re} \frac{1}{1+d}$. Thus $c=s b$. This conclusion also holds when $\varphi$ is an affine map, $\varphi(z)=\eta(s z+1-s)$, where the computations are easier.

For the final statement, let $\psi=\rho_{\eta,-b} \circ \varphi$, so that $\psi$ is an automorphism of $\mathbb{D}$ and $\varphi=\rho_{\eta, b} \circ \psi$. Since the Krein adjoint of an automorphism is its inverse, we have

$$
\sigma=\sigma_{\varphi}=\sigma_{\psi} \circ \sigma_{\rho_{\eta, b}}=\psi^{-1} \circ \rho_{\eta, \bar{b}}=\psi^{-1} \circ \rho_{\eta, b}
$$

and thus $\varphi \circ \sigma=\rho_{\eta, b} \circ \psi \circ \psi^{-1} \circ \rho_{\eta, b}=\rho_{\eta, 2 b}$. Similarly, $\sigma \circ \varphi=\rho_{1,2 c}=\rho_{\zeta, 2 c}$.

The remarks preceeding Theorem [6 express the linear-fractional maps $\psi$ with $C_{\psi}$ belonging to $C^{*}\left(C_{\varphi}, \mathcal{K}\right)$ in terms of $\varphi, \sigma, \rho_{\eta, a}$ and $\rho_{\zeta, a}$ for appropriate ranges of the translation numbers $a$. We describe below a corresponding operator-theoretic description of $C_{\psi}$ modulo $\mathcal{K}$, in terms of the polar factors of $C_{\varphi}$ and $C_{\varphi}^{*}$. In [15] it was shown that every operator $B$ in $C^{*}\left(C_{\varphi}, \mathcal{K}\right)$ has a representation generalizing Equation (2) and having the form $B=T+K$ with $K$ compact and

$$
T=c I+f\left(C_{\varphi}^{*} C_{\varphi}\right)+g\left(C_{\varphi} C_{\varphi}^{*}\right)+U h\left(C_{\varphi}^{*} C_{\varphi}\right)+U^{*} k\left(C_{\varphi} C_{\varphi}^{*}\right),
$$

where $f$ and $h$ are continuous on $\sigma\left(C_{\varphi}^{*} C_{\varphi}\right), g$ and $k$ are continuous on $\sigma\left(C_{\varphi} C_{\varphi}^{*}\right)$, all four functions vanish at zero, and $U$ is the partial isometry polar factor (which in this case is unitary) of $C_{\varphi}$. The restrictions of $f, g, h$ and $k$ to the interval [0,s], which coincides with both of the essential spectra $\sigma_{e}\left(C_{\varphi}^{*} C_{\varphi}\right)$ and $\sigma_{e}\left(C_{\varphi} C_{\varphi}^{*}\right)$, are uniquely determined by $B$. We call $T$ a distinguished representative of the coset $[B]$, and recall from [15] that

$$
\Psi(B)=\Psi(T)=\left[\begin{array}{lr}
c+g & h \\
k & c+f
\end{array}\right] .
$$

We start with the operator $\left(C_{\varphi}^{*} C_{\varphi}\right)^{a}$, defined by the self-adjoint functional calculus, where Re $a>0$. Note that

$$
C_{\varphi}^{*} C_{\varphi} \equiv s C_{\sigma} C_{\varphi}(\bmod \mathcal{K})=s C_{\varphi \circ \sigma}=s C_{\rho_{\eta, 2 b}}
$$


where the last equality follows from Theorem 6. In Theorem 3. take $\gamma=\eta$ and consider the $*$-isomorphism $\Gamma$, here called $\Gamma_{\eta}$ to emphasize the fixed point $\eta$. We have

$$
\left[\left(C_{\varphi}^{*} C_{\varphi}\right)^{a}\right]=\left[C_{\varphi}^{*} C_{\varphi}\right]^{a}=s^{a}\left[C_{\rho_{\eta, 2 b}}\right]^{a}=s^{a} \Gamma_{\eta}\left(t^{2 b}\right)^{a}=s^{a} \Gamma_{\eta}\left(t^{2 b a}\right)=s^{a}\left[C_{\rho_{\eta, 2 b a}}\right] .
$$

The first and fourth equalities follow, respectively, from the facts that the coset map $B \mapsto[B]$ and $\Gamma_{\eta}$ are each $*$-homomorphisms. Relabeling $2 b a$ as $a$, we see that $s^{-\frac{a}{2 b}}\left(C_{\varphi}^{*} C_{\varphi}\right)^{\frac{a}{2 b}}$ is a distinguished representative of $\left[C_{\rho_{\eta, a}}\right]$ for Re $a>0$. A similar argument shows that the coset $\left[C_{\rho_{\zeta, a}}\right]$ has distinguished representative $s^{-\frac{a}{2 c}}\left(C_{\varphi} C_{\varphi}^{*}\right)^{\frac{a}{2 c}}$ for $\operatorname{Re} a>0$.

Now consider $\rho_{\eta, a} \circ \varphi$, which we know to be a self-map of $\mathbb{D}$, but not an automorphism, when Re $a>-b$. First we look at the case Re $a>0$. We have

$$
C_{\rho_{\eta, a} \circ \varphi}=C_{\varphi} C_{\rho_{\eta, a}}=U\left(C_{\varphi}^{*} C_{\varphi}\right)^{\frac{1}{2}} C_{\rho_{\eta, a}} \equiv s^{-\frac{a}{2 b}} U\left(C_{\varphi}^{*} C_{\varphi}\right)^{\frac{1}{2}+\frac{a}{2 b}}(\bmod \mathcal{K})
$$

by our above discussion. By the spectral theorem, $\left(C_{\varphi}^{*} C_{\varphi}\right)^{z}$ is holomorphic for Re $z>0$ in the weak operator topology, and therefore in the operator norm topology; see [11], Theorem 3.10.1. Thus the cosets $\left[C_{\rho_{\eta, a} \circ \varphi}\right]$ and $\left[s^{-\frac{a}{2 b}} U\left(C_{\varphi}^{*} C_{\varphi}\right)^{\frac{1}{2}+\frac{a}{2 b}}\right]$ are both holomorphic $\mathcal{B}\left(H^{2}\right) / \mathcal{K}$-valued functions of $a$, Re $a>-b$, which agree on the subset $\{a: \operatorname{Re} a>0\}$. Hence they agree on all of $\{a: \operatorname{Re} a>-b\}$, showing that $s^{-\frac{a}{2 b}} U\left(C_{\varphi}^{*} C_{\varphi}\right)^{\frac{1}{2}+\frac{a}{2 b}}$ is a distinguished representative of $\left[C_{\rho_{\eta, a} \circ \varphi}\right]$ when $\operatorname{Re} a>-b$. An analogous statement holds for $\left[C_{\rho_{\zeta, a} \circ \sigma}\right]$ with Re $a>-c$. The following table

\begin{tabular}{|c|c|c|c|}
\hline \multicolumn{4}{|c|}{ TABLE II } \\
\hline \multicolumn{4}{|c|}{ Linear-fractional $\psi$ with $C_{\psi}$ in $C^{*}\left(C_{\varphi}, \mathcal{K}\right)$} \\
\hline $\begin{array}{l}\text { Condition on } \psi \\
\text { in Theorem } 5]\end{array}$ & $\psi$ & $\begin{array}{l}\text { Distinguished } \\
\text { representative of }\left[C_{\psi}\right]\end{array}$ & $\begin{array}{c}\text { Matrix function } \\
\Psi\left(C_{\psi}\right)(t), 0 \leq t \leq s\end{array}$ \\
\hline (d) & $\begin{array}{c}\rho_{\eta, a} \\
\operatorname{Re} a>0\end{array}$ & $s^{-\frac{a}{2 b}}\left(C_{\varphi}^{*} C_{\varphi}\right)^{\frac{a}{2 b}}$ & {$\left[\begin{array}{cc}\left(\frac{t}{s}\right)^{\frac{a}{2 b}} & 0 \\
0 & 0\end{array}\right]$} \\
\hline (b) & $\begin{array}{c}\rho_{\zeta, a}, \\
\operatorname{Re} a>0\end{array}$ & $s^{-\frac{a}{2 c}}\left(C_{\varphi} C_{\varphi}^{*}\right)^{\frac{a}{2 c}}$ & {$\left[\begin{array}{cc}0 & 0 \\
0 & \left(\frac{t}{s}\right)^{\frac{a}{2 c}}\end{array}\right]$} \\
\hline (a) & $\begin{array}{c}\rho_{\eta, a} \circ \varphi \\
\operatorname{Re} a>-b\end{array}$ & $s^{-\frac{a}{2 b}} U\left(C_{\varphi}^{*} C_{\varphi}\right)^{\frac{1}{2}+\frac{a}{2 b}}$ & {$\left[\begin{array}{cc}0 & \sqrt{t}\left(\frac{t}{s}\right)^{\frac{a}{2 b}} \\
0 & 0\end{array}\right]$} \\
\hline (c) & $\begin{array}{c}\rho_{\zeta, a} \circ \sigma, \\
\operatorname{Re} a>-c\end{array}$ & $s^{-\frac{a}{2 c}-1} U^{*}\left(C_{\varphi} C_{\varphi}^{*}\right)^{\frac{1}{2}+\frac{a}{2 c}}$ & {$\left[\begin{array}{cc}0 & 0 \\
\frac{\sqrt{t}}{s}\left(\frac{t}{s}\right)^{\frac{a}{2 c}} & 0\end{array}\right]$} \\
\hline
\end{tabular}
summarizes these conclusions.

Given an operator $B$ in $C^{*}\left(C_{\varphi}, \mathcal{K}\right), \sigma_{e}(B)$ and $\|B\|_{e}$ coincide with $\sigma(\Psi(B))$ and $\|\Psi(B)\|$, respectively. Thus, if $B$ is a finite linear combination of composition operators $C_{\psi}$ with $\psi$ 's chosen from Column 2 in Table II, one can calculate $\Psi(B)$ from Column 4 and in principle read off $\sigma_{e}(B)$ and $\|B\|_{e}$; see Theorem 4.17 in [15].

It is known [14 that the collection of linear-fractional composition operators $C_{\psi}$ with $\psi$ a non-automorphism having $\|\psi\|_{\infty}=1$ is linearly independent modulo $\mathcal{K}$. The following result shows that this remains true when $\mathcal{K}$ is replaced by the larger subspace $C^{*}\left(C_{\varphi}, \mathcal{K}\right)$ of $\mathcal{B}\left(H^{2}\right)$. 
Theorem 7. Let $\varphi$ be as in (11). Suppose that $\beta_{1}, \cdots, \beta_{q}$ are distinct linearfractional self-maps of $\mathbb{D}$ and that $a_{1} \cdots, a_{q}$ are non-zero complex numbers. If the linear combination $a_{1} C_{\beta_{1}}+\cdots+a_{q} C_{\beta_{q}}$ lies in $C^{*}\left(C_{\varphi}, \mathcal{K}\right)$, then so do the individual operators $C_{\beta_{1}}, \cdots, C_{\beta_{q}}$.

Proof. Let us discard those $C_{\beta_{i}}$ 's which lie in $C^{*}\left(C_{\varphi}, \mathcal{K}\right)$ and assume for the purpose of obtaining a contradiction that there are some left over. Relabel these as $C_{\beta_{1}}, \cdots, C_{\beta_{r}}$, let $a_{1}, \cdots, a_{r}$ be the corresponding constants, and put $T=a_{1} C_{\beta_{1}}+$ $\cdots+a_{r} C_{\beta_{r}}$, which lies in $C^{*}\left(C_{\varphi}, \mathcal{K}\right)$. Here, none of the summands are compact, so $\left\|\beta_{i}\right\|_{\infty}=1$ for $i=1, \cdots, r$. Now we proceed almost as in the proof of Theorem 1 with $T$ playing the role of $C_{\psi}$. Given $\epsilon>0$ there exists $A$ as in that proof and a complex $c$ such that $\left\|T-c C_{z}-A\right\|_{e}<\epsilon$. By the inequality (11)

$$
\left\|T-c C_{z}-A\right\|_{e}^{2} \geq|c|^{2}+\frac{1}{2 \pi} \sum_{i=1}^{r}\left|a_{i}\right|^{2}\left|J\left(\beta_{i}\right)\right|
$$

so that $|c|<\epsilon$, since each $\left|J\left(\beta_{i}\right)\right|$ must be zero, so $\beta_{i}$ is a non-automorphism. As earlier, we have $\|T-A\|_{e}<2 \epsilon$.

According to Corollary 5.17 in [14, the cosets $\left[C_{\beta_{1}}\right], \cdots,\left[C_{\beta_{r}}\right]$ are linearly independent in $\mathcal{B}\left(H^{2}\right) / \mathcal{K}$. It follows that $T$ is not compact, so the matrix function

$$
\Psi(T)=\left[\begin{array}{ll}
f_{2} & f_{3} \\
f_{4} & f_{1}
\end{array}\right]
$$

is not identically zero on $[0, s]$. As in Theorem 1, we focus on $f_{3}$ and aim for a contradiction by assuming that $\left\|f_{3}\right\|_{\infty}>0$. An appropriate choice of $\epsilon$ again yields the inequality (12), where $p$ has the same meaning as there. Again we write $A$ in the form (13), and thus have

$$
\begin{aligned}
4 \epsilon^{2}>\|T-A\|_{e}^{2} & \geq \limsup _{|z| \rightarrow 1}\left\|\left(T^{*}-A^{*}\right) \frac{k_{z}}{\left\|k_{z}\right\|}\right\|^{2} \\
& \geq \lim _{\Gamma_{\zeta, D}}\left\|\left(\sum_{i=1}^{r} \overline{a_{i}} C_{\beta_{i}}^{*}-\sum_{i=1}^{m} \overline{c_{i}} C_{\psi_{i}}^{*}\right) \frac{k_{z}}{\left\|k_{z}\right\|}\right\|^{2} \\
& \geq\left\|\sum_{\substack{\zeta \in F\left(\psi_{i}\right) \\
D_{1}\left(\psi_{i}, \zeta\right)=\mathbf{d}_{3}}} \overline{c_{i}} k_{w_{i}}^{+}\right\|_{H_{+}^{2}}^{2}
\end{aligned}
$$

The rest of the argument follows that of Theorem 1 exactly, reaching the same contradiction.

\section{NON LINEAR-FRACTIONAL MAPS}

In this section we explore maps $\psi$, satisfying either condition (e) or (f) of Theorem 2, for which $C_{\psi}$ lies in $C^{*}\left(C_{\varphi}, \mathcal{K}\right)$; our main result shows that such maps exist. We begin with a lemma about finite Blaschke products.

Lemma 5. Given $\zeta, \eta$ distinct points on $\partial \mathbb{D}$, and positive numbers $t_{1}, t_{2}$, there exists a finite Blaschke product $B$ with the properties $B(\eta)=\eta, B(\zeta)=\eta, B^{\prime}(\eta)=t_{1}$ and $\left|B^{\prime}(\zeta)\right|=t_{2}$. Moreover, $B^{\prime}(\zeta)=\eta \bar{\zeta} t_{2}$. 
Proof. Clearly there is no loss of generality in taking $\eta=1$. Initially we will also suppose that $\zeta=-1$; this condition will be removed at the end. A finite Blaschke product $B(z)=\prod \frac{\left|a_{n}\right|}{a_{n}}\left(a_{n}-z\right) /\left(1-\overline{a_{n}} z\right)$ will meet the conditions $B(1)=1$, $B(-1)=1$ if both

$$
\prod \frac{\left|a_{n}\right|}{a_{n}} \frac{a_{n}-1}{1-\overline{a_{n}}}=1
$$

and

$$
\prod \frac{\left|a_{n}\right|}{a_{n}} \frac{a_{n}+1}{1+\overline{a_{n}}}=1
$$

It is easy to see that both of these conditions will be met if the zeros of $B$ are chosen to be a collection of conjugate pairs $\{a, \bar{a}\}$. The conditions $B^{\prime}(1)=t_{1},\left|B^{\prime}(-1)\right|=t_{2}$ are satisfied if

$$
\sum \frac{1-\left|a_{n}\right|^{2}}{\left|1-a_{n}\right|^{2}}=t_{1}
$$

and

$$
\sum \frac{1-\left|a_{n}\right|^{2}}{\left|1+a_{n}\right|^{2}}=t_{2}
$$

respectively (see [2]).

Next observe that for any $t>0,\left\{z: 1-|z|^{2}=t|1-z|^{2}\right\}$ is a circle centered at $(t /(t+1), 0)$ with radius $1 /(t+1)$ and $\left\{z: 1-|z|^{2}=t|1+z|^{2}\right\}$ is a circle centered at $(-t /(t+1), 0)$ with radius $1 /(1+t)$. As $t \rightarrow 0$, the centers of these circles approach 0 and the radii tend to 1 . Thus given $t_{1}, t_{2}$ arbitrary positive numbers we may choose $m$ a positive integer sufficiently large so that the circles $\left\{z: 1-|z|^{2}=\frac{t_{1}}{2^{m}}|1-z|^{2}\right\}$ and $\left\{z: 1-|z|^{2}=\frac{t_{2}}{2^{m}}|1+z|^{2}\right\}$ intersect in a conjugate pair of points $a, \bar{a}$. Consider the Blaschke product $B(z)$ with a zero of order $2^{m-1}$ at $a$ and a zero of order $2^{m-1}$ at $\bar{a}$. Since the zeros occur at conjugate pairs, $B(1)=1$ and $B(-1)=1$. By construction

$$
\left(1-|a|^{2}\right) /\left(|1-a|^{2}\right)=\left(1-|\bar{a}|^{2}\right) /\left(|1-\bar{a}|^{2}\right)=\frac{t_{1}}{2^{m}},
$$

and

$$
\left(1-|a|^{2}\right) /\left(|1+a|^{2}\right)=\left(1-|\bar{a}|^{2}\right) /\left(|1+\bar{a}|^{2}\right)=\frac{t_{2}}{2^{m}},
$$

so that the zeros of $B$ satisfy Equations (24) and (25) as desired, and $B^{\prime}(1)=t_{1}$, $\left|B^{\prime}(-1)\right|=t_{2}$.

Now suppose $\zeta \in \partial \mathbb{D}$ is not equal to -1 . Find a parabolic automorphism $\tau$ fixing 1 , with derivative 1 there, and taking $\zeta$ to -1 ; a unique such $\tau$ exists since (purely imaginary) translations act transitively on the boundary of the right half-plane. Then for $B$ as constructed above, $B \circ \tau$ is a finite Blaschke product fixing 1, with derivative $t_{1}$ at 1 , sending $\zeta$ to 1 and having derivative $\left|(B \circ \tau)^{\prime}(\zeta)\right|=\left|B^{\prime}(-1)\right|\left|\tau^{\prime}(\zeta)\right|$; since $\left|B^{\prime}(-1)\right|$ can be arbitarity prescribed and $\tau$ depends only on the value of $\zeta$, this means $\left|(B \circ \tau)^{\prime}(\zeta)\right|$ can be chosen to be an arbitrary positive number. Finally observe that if $B$ is a Blaschke product with $B(\zeta)=\eta$ and $\left|B^{\prime}(\zeta)\right|=s$, then we must have $B^{\prime}(\zeta)=\eta \bar{\zeta} s$, since $\bar{\eta} \zeta B(z)$ fixes $\zeta$, and hence has positive derivative there.

Theorem 8. Suppose that $\varphi$ is as in (1). There exist analytic self-maps $\psi_{1}$ and $\psi_{2}$ of $\mathbb{D}$, satifying conditions $(e)$ and $(f)$ of Theorem 0 respectively, such that $C_{\psi_{1}}$ and 
$C_{\psi_{2}}$ lie in $C^{*}\left(C_{\varphi}, \mathcal{K}\right)$. Moreover, $\psi_{1}$ and $\psi_{2}$ can be taken to extend continuously to the closed disk $\overline{\mathbb{D}}$.

Proof. We shall indicate the construction for $\psi_{1}$ with the normalization $\eta=1$. First consider a simply connected domain $\Omega$ in $\mathbb{D}$, whose boundary is a smooth Jordan curve which at 1 and at -1 includes an arc of an internally tangent circle, such that $\bar{\Omega} \cap \partial \mathbb{D}=\{-1,1\}$. It is easy to construct a conformal map $\rho: \mathbb{D}$ onto $\Omega$, extending to a homeomorphism of $\bar{D}$ onto $\bar{\Omega}$, with the properties $\rho(1)=1, \rho(-1)=-1$ and $\rho^{\prime}(1)=\frac{1}{2}$. The map $\rho$ will be analytic in a neighborhood of both 1 and -1 . Let $\tau$ be the unique parabolic automorphism of $\mathbb{D}$ fixing 1 , having derivative 1 there, and mapping $\zeta$ to -1 . By the uniqueness statement, $\tau^{\prime}(\zeta)$ is determined by $\zeta$. Using Lemma 5] construct a finite Blaschke product $B$ with $B(1)=1, B(-1)=1, B^{\prime}(1)=$ 2 and $\left|B^{\prime}(-1)\right|$ chosen so that $\left|B^{\prime}(-1)\right|\left|\rho^{\prime}(-1)\right|\left|\tau^{\prime}(\zeta)\right|=s$, where $s=\left|\varphi^{\prime}(\zeta)\right|$. The map $\psi_{1} \equiv B \circ \rho \circ \tau$ is a self-map of $\mathbb{D}$, with finite angular derivative set $F\left(\psi_{1}\right)=\{\zeta, 1\}$ and satisfying $\psi_{1}(\zeta)=1, \psi_{1}(1)=1, \psi_{1}^{\prime}(1)=1$ and $\psi_{1}^{\prime}(\zeta)=\bar{\zeta} s$, this last condition following from $\left|\psi_{1}^{\prime}(\zeta)\right|=s$ and $\psi_{1}(\zeta)=1$. Clearly $\psi_{1}$ has order of contact two at 1 and $\zeta$.

Any linear-fractional map $\beta$ is uniquely determined by its second order data vector $D_{2}\left(\beta, z_{0}\right)=\left(\beta\left(z_{0}\right), \beta^{\prime}\left(z_{0}\right), \beta^{\prime \prime}\left(z_{0}\right)\right)$ at any point $z_{0}$ of analyticity. The curvature of the curve $\psi_{1}(\partial \mathbb{D})$ at the points $\psi_{1}(\zeta)$ and $\psi_{1}(1)$ is determined by $D_{2}\left(\psi_{1}, \zeta\right)$ and $D_{2}\left(\psi_{1}, 1\right)$, respectively. By construction of $\psi_{1}$, these curvatures exceed unity. There exists unique linear-fractional maps $\beta_{1}, \beta_{2}$ of $\mathbb{D}$ with the second order data vectors

$$
D_{2}\left(\beta_{1}, 1\right)=D_{2}\left(\psi_{1}, 1\right)=\left(1,1, \psi_{1}^{\prime \prime}(1)\right) \text { and } D_{2}\left(\beta_{2}, \zeta\right)=D_{2}\left(\psi_{1}, \zeta\right)=\left(1, \bar{\zeta} s, \psi_{1}^{\prime \prime}(\zeta)\right) \text {. }
$$

The curvature of $\left\{\psi_{1}\left(e^{i \theta}\right): e^{i \theta} \in \partial \mathbb{D}\right\}$ matches that of $\beta_{1}(\partial \mathbb{D})$ at $e^{i \theta}=1$ and that of $\beta_{2}(\partial \mathbb{D})$ at $e^{i \theta}=\zeta$. Thus $\beta_{1}$ and $\beta_{2}$ are non-automorphism self-maps of $\mathbb{D}$. Since $\varphi^{\prime}(\zeta)=\psi_{1}^{\prime}(\zeta)=\beta_{2}^{\prime}(\zeta)$, Theorem 5 shows that $C_{\beta_{1}}$ and $C_{\beta_{2}}$ are in $C^{*}\left(C_{\varphi}, \mathcal{K}\right)$, and hence so is $C_{\beta_{1}}+C_{\beta_{2}}$. By Corollary 5.16 of [14], $C_{\psi_{1}} \equiv C_{\beta_{1}}+C_{\beta_{2}}(\bmod \mathcal{K})$ and thus $C_{\psi_{1}}$ is in $C^{*}\left(C_{\varphi}, \mathcal{K}\right)$.

For $\psi_{2}$, note that the Krein adjoint $\sigma$ of $\varphi$ satisfies $\sigma(\eta)=\zeta$ and $\sigma^{\prime}(\eta)=1 / \varphi^{\prime}(\zeta)$. We apply the first part of the proof, with $\varphi$ replaced by $\sigma$, to find a self map $\psi_{2}$ of $\mathbb{D}$ with $F\left(\psi_{2}\right)=\{\zeta, \eta\}, \psi_{2}(\eta)=\zeta, \psi_{2}^{\prime}(\eta)=\sigma^{\prime}(\eta)=1 / \varphi^{\prime}(\zeta), \psi_{2}(\zeta)=\zeta$, and $\psi_{2}^{\prime}(\zeta)=1$ such that $C_{\psi_{2}} \in C^{*}\left(C_{\sigma}, \mathcal{K}\right)=C^{*}\left(C_{\varphi}, \mathcal{K}\right)$ as desired.

Our last theorem shows that for sufficiently nice $\psi$, Theorem 2 is almost the whole story.

Theorem 9. Let $\psi$ be an analytic self-map of $\mathbb{D}$ such that $F(\psi)$ is a finite set, $\psi$ extends analytically to a neighborhood of each point in $F(\psi)$, and for any open set $U$ of $\partial \mathbb{D}$ containing $F(\psi),\left\|\chi_{\partial \mathbb{D} \backslash U} \psi\right\|_{\infty}<1$. If $\varphi$ is as in (1), then $C_{\psi}$ lies in $C^{*}\left(C_{\varphi}, \mathcal{K}\right)$ if and only if

(i) one of the conditions (a)-(f) of Theorem 0 holds, and

(ii) the map $\psi$ has order of contact two at each point of $F(\psi)$.

Proof. Suppose $\psi$ is as described and $C_{\psi}$ is in $C^{*}\left(C_{\varphi}, \mathcal{K}\right)$. By Theorem 2, one of (a)-(f) holds. Suppose that $\gamma$ is in $F(\psi)$ and $\psi$ has order of contact exceeding two at $\gamma$. Following Theorem 1, we let $\epsilon>0$ and find a finite linear combination $A$ of composition operators whose self-maps are chosen from the lists (6) such that $\left\|C_{\psi}-A\right\|<\epsilon$. At the same time we apply the inequality (9) to the linear 
combination $C_{\psi}-A$ at the point $\alpha=\gamma$. The maps in the lists (6), being linearfractional non-automorphisms, all have order of contact two at the unique points in their angular derivative sets. Taking the left side of (9) to be $\left\|C_{\psi}-A\right\|_{e}^{2}$ and $k=3$ on the right side, the sum on the right-hand side has only one term, namely $1 /\left|\psi^{\prime}(\gamma)\right|$, giving

$$
\epsilon^{2}>\left\|C_{\psi}-A\right\|_{e}^{2} \geq \frac{1}{\left|\psi^{\prime}(\gamma)\right|}
$$

a contradiction. Thus $\psi$ must have order of contact two at $\gamma$.

Conversely, suppose $\psi$ satisfies (i) and (ii). If $F(\psi)=\{\zeta\}$, let $\beta$ be the unique linear-fractional map with $D_{2}(\beta, \zeta)=D_{2}(\psi, \zeta)$. Since $\psi$ has order of contact two at $\zeta$, the curvature of $\left\{\psi\left(e^{i \theta}\right): e^{i \theta} \in \partial \mathbb{D}\right\}$ at $e^{i \theta}=\zeta$ exceeds unity, so that $\beta(\partial \mathbb{D})$, having the same curvature, is internally tangent to $\partial \mathbb{D}$ at $\psi(\zeta)$ and bounds a proper subdisk of $\mathbb{D}$. Thus $\beta$ is a non-automorphism of $\mathbb{D}$ satisfying (a) or (b) of Theorem 5 . and $C_{\beta}$ lies in $C^{*}\left(C_{\varphi}, \mathcal{K}\right)$. The same argument covers the case $F(\psi)=\{\eta\}$.

If $F(\psi)=\{\zeta, \eta\}$ we proceed as in the proof of Theorem 8 to produce linearfractional non-automorphisms $\beta_{1}$ and $\beta_{2}$ of $\mathbb{D}$ with $C^{*}\left(C_{\varphi}, \mathcal{K}\right)$ containing $C_{\beta_{1}}$ and $C_{\beta_{2}}$ and $C_{\psi} \equiv C_{\beta_{1}}+C_{\beta_{2}}(\bmod \mathcal{K})$.

\section{REFERENCES}

[1] P. Bourdon, D. Levi, S. Narayan and J. Shapiro, Which linear fractional composition operators are essentially normal?, J. Math. Anal. Appl. 280 (2003), 30-53.

[2] P. Ahern and D. Clark, On inner functions with $H^{p}$-derivative, Michigan Math. J. 21 (1974), $115-127$.

[3] E. Basor and D. Retsek, Extremal non-compactness of composition operators with linear fractional symbol, J. Math. Anal. Appl. 322 (2006), 749-763.

[4] E. Berkson, Composition operators isolated in the uniform operator topology, Proc. Amer. Math. Soc. 81 (1981), 230-232.

[5] J. Conway, A Course in Operator Theory, Amer. Math. Soc., Providence, 2000.

[6] C. Cowen, Composition operators on $H^{2}$, J. Operator Theory 9 (1983), 77-106.

[7] C. Cowen, Linear-fractional composition operators on $H^{2}$, Integral Equations Operator Theory 11 (1988), 151-160.

[8] C. Cowen and B. MacCluer, Composition Operators on Spaces of Analytic Functions, CRC Press, Boca Raton, 1995.

[9] A. Dash, Joint essential spectra, Pacific J. Math., 64 (1976), 119-128.

[10] J. Guyker, On reducing subspaces of composition operators, Acta Sci. Math. (Szeged) 53 (1989), 369-376.

[11] E. Hille and R. Phillips, Functional Analysis and Semi-Groups, Amer. Math. Soc., Providence, 1957.

[12] M. Jury, $C^{*}$-algebras generated by groups of composition operators, Indiana Univ. Math. J. 56 (2007), 3171-3192.

[13] M. Jury, The Fredholm index for elements of Toeplitz-composition $C^{*}$-algebras, Integral Equations Operator Theory, 58 (2007), 341-362.

[14] T. Kriete and J. Moorhouse, Linear relations in the Calkin algebra for composition operators, Trans. Amer. Math. Soc. 359 (2007), 2915-2944.

[15] T. Kriete, B. MacCluer, and J. Moorhouse, Toeplitz-composition $C^{*}$-algebras, J. Operator Theory 58 (2007), 135-156.

[16] T. Kriete, B. MacCluer, and J. Moorhouse, Spectral theory for algebraic combinations of Toeplitz and composition operators, preprint.

[17] B. MacCluer and J. Shapiro, Angular derivatives and compact composition operators on the Hardy and Bergman spaces, Canadian J. Math. 38 (1986), 878-906.

[18] A. Montes-Rodríguez, M. Ponce-Escudero, and S. Shkarin, Invariant subspaces of parabolic self-maps in the Hardy space, preprint.

[19] J. Shapiro and C. Sundberg, Isolation amongst the composition operators, Pacific J. Math. 145 (1990), 117-152. 
[20] J. Shapiro and P. Taylor, Compact, nuclear, and Hilbert-Schmidt composition operators on $H^{2}$, Indiana Univ. Math. J. 23 (1973), 471-496.

[21] J. Shapiro, Composition Operators and Classical Function Theory, Springer-Verlag, New York, 1993.

Department of Mathematics, University of Virginia, Charlottesville, VA 22904

E-mail address: tlk8q@virginia.edu

Department of Mathematics, University of Virginia, Charlottesville, VA 22904

E-mail address: bdm3f@virginia.edu

Department of Mathematics, Colgate University, Hamilton, NY 13356

E-mail address: jmoorhouse@mail.colgate.edu 